\title{
Optimizing force spectroscopy by modifying commercial cantilevers: improved stability, precision, and temporal resolution
}

\author{
Devin T. Edwards ${ }^{a}$ and Thomas T. Perkins ${ }^{a, b,{ }^{*}}$
}


ABSTRACT Atomic force microscopy (AFM)-based single-molecule force spectroscopy (SMFS) enables a wide array of studies, from measuring the strength of a ligand-receptor bond to elucidating the complex folding pathway of individual membrane proteins. Such SMFS studies and, more generally, the diverse applications of AFM across biophysics and nanotechnology are improved by enhancing data quality via improved force stability, force precision, and temporal resolution. For an advanced, small-format commercial AFM, we illustrate how these three metrics are limited by the cantilever itself rather than the larger microscope structure, and then describe three increasingly sophisticated cantilever modifications that yield enhanced data quality. First, sub-pN force precision and stability over a broad bandwidth $(\Delta f=0.01-20 \mathrm{~Hz})$ is routinely achieved by removing a long $(L=100 \mu \mathrm{m})$ cantilever's gold coating. Next, this sub-pN bandwidth is extended by a factor of $\sim 50$ to span five decades of bandwidth $(\Delta f=0.01-1,000$ $\mathrm{Hz}$ ) by using a focused ion beam (FIB) to modify a shorter $(L=40 \mu \mathrm{m})$ cantilever. Finally, FIBmodifying an ultrashort $(L=9 \mu \mathrm{m})$ cantilever improves its force stability and precision while maintaining 1- $\mu \mathrm{s}$ temporal resolution. These modified ultrashort cantilevers have a reduced quality factor $(Q \approx 0.5)$ and therefore do not apply a substantial $(30-90 \mathrm{pN})$, high-frequency force modulation to the molecule, a phenomenon that is unaccounted for in traditional SMFS analysis. Currently, there is no perfect cantilever for all applications. Optimizing AFM-based SMFS requires understanding the tradeoffs inherent to using a specific cantilever and choosing the one best suited to a particular application.

Keywords: atomic force microscopy; protein folding; single-molecule force spectroscopy; singlemolecule biophysics; focused-ion-beam modification; cantilever dynamics.

Abbreviations: AFM, atomic force microscopy; FIB, focused ion beam; PSD, power spectral density; SEM, scanning electron microscopy; SMFS, single-molecule force spectroscopy, WLC, worm-like chain. 


\section{Introduction}

Advanced physical techniques now allow one to pull on individual biomolecules with sub-pN force precision (Greenleaf et al., 2007; Neuman and Nagy, 2008) and to push on sub-cellular regions of single cells with nanometer-scale lateral resolution (Scheuring and Dufrene, 2010). This wide-ranging field, called force spectroscopy, provides insights to important biological questions over a broad spectrum of biological systems and length scales. Single-molecule force spectroscopy (SMFS) has proven particularly powerful. In this field, individual molecules of DNA are stretched (Smith et al., 1992), allowing diverse DNA-based molecular motors to be studied (Chemla, 2010; Herbert et al., 2008). In other work, the robust streptavidin-biotin bond is dissociated (Florin et al., 1994; Lee et al., 1994a), elucidating fundamental aspects of thermally activated bond rupture under load (Evans and Ritchie, 1997; Merkel et al., 1999). Finally, the unfolding and refolding of individual protein domains (Rief et al., 1997) and nucleicacid structures (Liphardt et al., 2001) is a particularly exciting application that enables the detection of folding intermediates that are unresolved in ensemble assays (Cecconi et al., 2005; Stigler et al., 2011; Yu et al., 2012b). SMFS even allows researchers to reconstruct a 1dimensional projection of the folding-energy landscape (Woodside et al., 2006b), providing experimental access to the central unifying concept in protein folding (Onuchic et al., 1997). Improving the quality of the underlying data enhances all of these exciting applications.

Atomic force microscopy (AFM)-based SMFS helped pioneer the mechanical unfolding of single proteins (Carrion-Vazquez et al., 1999; Marszalek et al., 1999; Oesterhelt et al., 2000; Rief et al., 1997), and remains a powerful tool for SMFS. More broadly, AFM has contributed to a wide range of biological studies, especially imaging (Muller and Dufrene, 2008). Given AFM's broad applicability, excellent reviews have discussed its use for imaging (Ando et al., 2013; Bippes and Muller, 2011), measuring the nanomechanical properties of materials ( $\mathrm{Li}$ and Cao, 2010; Zhang et al., 2014), investigating ligand binding (Dupres et al., 2007; Kienberger et al., 2006), and studying protein folding (Hoffmann and Dougan, 2012; Janovjak et al., 2008; Puchner and Gaub, 2009; Zoldak and Rief, 2013). Because the AFM cantilever plays a critical role in all these measurements, there has been increasing work in developing specialized cantilevers [e.g., torsionally oscillating cantilevers for rapid nanomechanical mapping (Sahin and Erina, 2008)]. In this article, we discuss improving the force stability, force precision, and 
temporal resolution of AFM-based SMFS by modifying commercially available cantilevers (Bull et al., 2014; Churnside et al., 2012; Edwards et al., 2015). The discussion is focused on proteinfolding studies where force stability is critical since the protein-unfolding rate varies exponentially with force $(F)$, whereas force precision $(\mathrm{pN} / \sqrt{ } \mathrm{Hz})$ and temporal resolution facilitate the detection of short-lived states. Collectively, improving these metrics via cantilever modifications should broadly benefit AFM-based studies.

In a typical AFM-based protein-unfolding assay, a polyprotein is stretched between a surface and the AFM tip (Fig. 1A). The force applied to the protein is deduced from cantilever deflection via a laser reflected off of a gold-coated cantilever. As the cantilever is withdrawn from the surface, the force on the molecule rises, increasing the probability that the protein under tension unfolds. In the simplest theoretical description of the unfolding process, the application of a constant $F$ lowers the height of the energy barrier $\left(\Delta G^{\ddagger}\right)$ at the transition state $\left(\Delta x^{\ddagger}\right)$ by $F \Delta x^{\ddagger}$, effectively "tilting" the folding-energy landscape (Fig. 1B) (Bell, 1978; Evans and Ritchie, 1997). When the protein unfolds, the sudden increase in the contour length $\left(L_{0}\right)$ causes an abrupt drop in F. A polyprotein contains multiple protein domains, and therefore its unfolding results in a force-extension curve that display a characteristic saw-tooth pattern, with each tooth corresponding to a domain unfolding (Fig. 1C) (Rief et al., 1997). In dynamic force spectroscopy, these unfolding events are measured as a function of loading rate $(\partial F / \partial t)$ and, for AFM-based SMFS, typically analyzed within the Bell-Evans model (Bell, 1978; Evans and Ritchie, 1997). This analysis yields $\Delta x^{*}$ and $k_{\text {off }}$, the unfolding rate at zero force. These oftmeasured parameters yield insight into the underlying folding-energy landscape.

Typically, AFM-based SMFS uses rapid-stretching protocols $(50-5,000 \mathrm{~nm} / \mathrm{s})$, in part to minimize limitations of the instrument. Unfortunately, such non-equilibrium studies yield a relatively small number of transitions per molecule. By contrast, equilibrium measurements with a state-of-the-art optical trap can monitor a single protein or nucleic acid structure unfolding and refolding thousands of times over hundreds of seconds (Stigler et al., 2011; Woodside et al., 2006a; Woodside et al., 2006b; Yu et al., 2012b). Such large data sets enable detailed studies of folding dynamics and reconstruction of folding-energy landscapes (Stigler et al., 2011; Woodside et al., 2006b). However, the observed folding probability is sensitive to sub-pN changes in $F$ (Liphardt et al., 2001), making exquisite force stability a prerequisite for such 
equilibrium studies. Limitations in the force stability of AFM have constrained analogous AFMbased measurements to near-equilibrium folding over several seconds (Junker et al., 2009). Hence, there is a clear need for enhanced force stability and precision in AFM to enable true equilibrium AFM-based studies.

SMFS studies of protein folding also benefit from increasing temporal resolution, which is critical to detecting short-lived ( $<1 \mathrm{~ms}$ ) intermediates (Stigler et al., 2011; Yu et al., 2012b). The transition path time - the fleeting time it takes to cross an activation barrier-occurs on even faster timescales, $\sim 1-10 \mu$ s for typical globular proteins (Chung et al., 2012; Lindorff-Larsen et al., 2011; Schuler and Hofmann, 2013). The temporal resolution in SMFS studies is ultimately limited by the mechanical response of the probe to a change in $F$ when immersed in liquid. For typical AFMs and optical traps, the response times are between 50-1,000 $\mu \mathrm{s}$, with advanced statistical techniques capable of probing the $10-\mu$ s regime (Zoldak et al., 2013). If the probe cannot respond to changes in $F$ significantly faster than the lifetime of the state, these states will be effectively filtered from the record. Such missing states prevent proper elucidation of the folding energy landscape. Thus, for AFM-based SMFS to have the largest impact, improvements in temporal resolution should accompany improvements in force precision and stability.

Recent developments of advanced, small-format AFMs have led to commercially available instruments where the tip-sample stability, detection sensitivity, and detector bandwidth do not limit the measurement. Rather, on such platforms, the limit of many applications is now the cantilever itself. To frame this discussion, we identify three timescales for the detected cantilever motion by analyzing the force power spectral density (PSD) of a cantilever immersed in liquid and positioned $50 \mathrm{~nm}$ over a surface (Fig. 1D). At low frequencies (Fig. 1D, purple shading), force noise increases rapidly with decreasing frequency. This instrumental noise arises from drift in the zero-force position $\left(z_{0}\right)$ of the cantilever and degrades performance at timescales as short as $100 \mathrm{~ms}$ for a gold-coated long BioLever $[L=100 \mu \mathrm{m} ; k=6 \mathrm{pN} / \mathrm{nm}$ (Olympus)] (Churnside et al., 2012). However, many force spectroscopy experiments collect data over times longer than $100 \mathrm{~ms}$, including both traditional dynamic force spectroscopy and the near-equilibrium assays discussed above. At slightly higher frequencies, the PSD is flat, showing that the cantilever motion is limited by the Brownian motion of the cantilever in liquid (Fig. 1D, peach shading). An early result from Viani et al. (1999) demonstrated that force precision in this regime is 
improved by using smaller cantilevers, which have lower hydrodynamic drag $(\beta)$. At still higher frequencies, cantilever motion falls off rapidly above the characteristic frequency $\left(f_{0}\right)$ (Fig. 1D, green shading). At these short timescales, the cantilever response lags the change in $F$, effectively filtering out information above $f_{0}$ and thereby hindering the detection of short-lived states and measurements of the transition path time. Thus, a complex interplay exists between cantilever properties and the quality of force spectroscopy measurements in terms of force stability, precision, and temporal resolution.

In this article, we outline a series of modifications to commercial cantilevers that can improve these three metrics in AFM-based SMFS (Bull et al., 2014; Churnside et al., 2012; Edwards et al., 2015). Three increasingly sophisticated modifications to commercial cantilevers each offer particular strengths and weaknesses, as summarized in Fig. 2. Specifically, we show that a simple modification - stripping the gold off a long, soft cantilever-yields sub-pN force stability and precision over an extended bandwidth $(0.01-10 \mathrm{~Hz})$ (Churnside et al., 2012). Such precision and stability are traditionally associated with optical traps and magnetic tweezers. By modifying a shorter cantilever using a focused ion beam (FIB), this sub-pN bandwidth was extended to cover five decades of bandwidth $(0.01-1,000 \mathrm{~Hz})$ while also retaining the cantilever's high reflectivity (Bull et al., 2014). We note that making such modifications is not overly complex; they are routinely done by undergraduates in our lab. Finally, we extended this concept to ultrashort $(L=9 \mu \mathrm{m})$ cantilevers. To optimize SMFS using ultrashort cantilevers on a commercial AFM, we FIB-modified these cantilevers and developed a home-built laser module capable of detecting them (Edwards et al., 2015). Taken together, these three cantilever modifications offer a means to significantly improve the quality of AFM-based SMFS. To understand how to best apply the different capabilities of these three cantilevers (Fig. 2), we first review how standard commercial cantilevers limit SMFS studies.

\section{Limitations to AFM-based force spectroscopy}

Many AFM users are familiar with the long time it takes an AFM to "settle" after loading a cantilever. Significant drift occurs even hours after loading a cantilever. Hence, a major focus of AFM manufacturers has been to build a stiffer AFM as a means to improve performance. While 
such improved mechanical designs perform impressively, they have not eliminated the need to let an AFM settle.

Our lab initially viewed this problem as one of tip-sample stability, meaning the tip was moving relative to the substrate. To improve tip-sample stability, we developed an optically stabilized AFM that held an AFM tip stationary to $0.3 \AA$ in $3 \mathrm{D}$ over $100 \mathrm{~s}$ and achieved a lateral tip-sample stability of $4 \AA$ over more than an hour (King et al., 2009). This optically stabilized AFM yielded a 100-fold improvement in tip-sample stability at ambient conditions. However, when we applied this AFM to stretch an individual protein at constant extension, we observed significant drift in the measured force despite the tip being actively stabilized with respect to the sample. The conceptual equivalent of this result is stretching a rubber band at constant extension and measuring a change in the force. Since the optically stabilized AFM assured us that the extension was indeed constant, the origin of the problem must reside in the process of measuring force. We went on to show that the primary source of the force drift was the cantilever's gold coating (Churnside et al., 2012).

\subsection{Gold-coated cantilevers have poor long-term force stability}

Force stability is critical to SMFS because the rate of macromolecular unfolding is exponentially sensitive to changes in $F$. The first step in isolating the gold coating as the source of the force drift was a simple experiment. We immersed a cantilever in liquid for $>2 \mathrm{~h}$, and then measured the cantilever's position $\left(z_{\text {tip }}\right)$ as a function of time. When no external force is applied, we refer to $z_{\text {tip }}$ as $z_{0}$, the zero-force position of the cantilever. This position should be constant. In contrast to this expectation, we measured a significant change in $z_{0}$ (Fig. 3A). The cantilever drifted by 7.5 nm over 5 min. To understand the detrimental effect of this drift on SMFS, we note that $F$ is deduced using $F=-k \Delta z=-k\left(z_{\text {tip }}-z_{0}\right)$, where $\Delta z$ is the change in tip deflection, and $z_{0}$ is assumed to be constant. Hence, this measured drift corresponds to a perceived change in $F$ of $540 \mathrm{pN}$ for the tested BioLever Mini, a cantilever promoted for SMFS applications. In a standard SMFS assay, this motion gets convolved in as a change in $F$ and, in a force clamp, it leads to an unintended and unmeasured change in the applied $F$. 
To highlight the need for force stability in SMFS, we show three traces of a DNA hairpin being stretched by an optical trap at a constant force around $F_{1 / 2}$, the force at which the hairpin is equally likely to be folded as unfolded (Fig. 3B). The data show that even $0.1 \mathrm{pN}$ changes in applied $F$ lead to measurable changes in the probability of being folded (Carter et al., 2009), a result similar to earlier work on nucleic acid structures (Liphardt et al., 2001; Woodside et al., 2006a) and proteins (Cecconi et al., 2005). Thus, it is clear that improving AFM's force stability is essential to improving the quality of the data and expanding the range of assays and systems suitable for study by AFM. Moreover, if the stability is sufficiently improved, AFMs could also perform true equilibrium assays where multiple cycles of unfolding and refolding are measured under constant external conditions, an exciting regime currently limited to optical-trapping (Cecconi et al., 2005; Liphardt et al., 2001; Stigler et al., 2011; Woodside et al., 2006a) and magnetic-tweezer-based assays (Chen et al., 2015; Kim and Saleh, 2009; Long et al., 2013). To date, AFM-based SMFS has achieved near-equilibrium studies where multiple transitions are observed, but not under stationary applied forces (He et al., 2015; Junker et al., 2009; Lee et al., 2010).

\subsection{Smaller cantilevers are not always better: low-frequency detection noise}

It is well established in AFM that smaller cantilevers are better for SMFS (Viani et al., 1999). The physical basis of this result is the fluctuation-dissipation theorem: $\Delta F=\sqrt{4 k_{\mathrm{B}} T \Delta f \beta}$, where $\Delta F$ is the force precision, $k_{B} T$ is the thermal energy, $\beta$ is the hydrodynamic drag of the cantilever, and $\Delta f$ is the bandwidth of the measurement. The primary way to reduce $\beta$ is to decrease cantilever length $(L)$. Hence, shorter cantilevers are better. However, an underlying assumption of this analysis is that the detected motion of the cantilever is limited by thermally induced Brownian motion rather than instrumental noise.

To investigate this assumption, we measured $z_{0}$ for two cantilevers of different lengths - the long BioLever $(L=100 \mu \mathrm{m} ; k=6 \mathrm{pN} / \mathrm{nm})$ and the BioLever Mini $(L=40 \mu \mathrm{m} ; k=100 \mathrm{pN} / \mathrm{nm})$ (Fig. 3C) - both of which are widely used in SMFS. We avoided the adverse effects of a cantilever's gold coating by chemically removing the gold and the underlying chromium layers from each cantilever (see §3.1) (Churnside et al., 2012). To further improve stability, we used a temperature-stabilized AFM (Cypher, Asylum Research) and measured the cantilevers $>2 \mathrm{~h}$ after 
immersion into liquid. As shown in Fig. 3D, the resulting positional PSDs recapitulate the information shown in Fig. 1D. At the lowest frequencies, the measured PSDs increase as the frequency decreases, a clear indication of instrumental noise. Over a limited middle-frequency range, each PSD was flat with respect to frequency, showing thermally limited response for an overdamped cantilever $(Q<0.5)$. The three primary differences between the two cantilevers in this thermally limited range are (i) the stiffer BioLever Mini has a lower positional noise, (ii) the BioLever Mini has a higher characteristic frequency $\left(f_{0}\right)$, and (iii) the thermally limited regime for the BioLever Mini covers a smaller frequency range. The first result is a consequence of the equipartition theorem, which states that stiffer cantilevers have less positional fluctuations: $\left\langle x^{2}\right\rangle$ $=k_{\mathrm{B}} T / k$. The second result follows from the fact that the BioLever Mini is stiffer and has reduced drag, which leads to a faster response time ( $\tau=\beta / k$ in the overdamped limit) and therefore a higher characteristic frequency. The potentially unexpected result is the third observation: the PSD of a BioLever Mini starts to deviate from a thermally limited response at 300-500 $\mathrm{Hz}(2-3$ $\mathrm{ms})$. We isolated the source of this instrumental noise by reflecting the detection laser off of the chip supporting the cantilever and scaling the resulting signal by the cantilever's sensitivity. Specifically, the low-frequency portion of the PSDs for both the long BioLever and the BioLever Mini becomes limited by this fixed instrumental noise $(\delta x)$ present in the optical-lever-arm detection system (Fig. 3D, black).

In force spectroscopy, the molecule responds to the force applied across it, so force rather than positional-based metrics are important. A particularly useful metric is the mean force precision over a given averaging time $(T)$. Technically, we use the Allan deviation, which is computed using $\sigma_{x}(T)=\sqrt{\frac{1}{2}\left\langle\left(\bar{x}_{\mathrm{i}+1}-\bar{x}_{\mathrm{i}}\right)^{2}\right\rangle_{\mathrm{T}}}$, where $\bar{x}_{i}$ is the mean value of the data over the $i^{\text {th }}$ time interval. The Allan deviation was developed to characterize atomic clocks (Sullivan et al., 1990) but is increasingly used to characterize instrumentational performance in single-molecule biophysics (Czerwinski et al., 2009). As shown in Fig. 3E, the force precision initially improves with increasing averaging time as $1 / \sqrt{T}$ for both cantilevers in the thermally limited region. The data also show that the force precision of the BioLever Mini is better than the long BioLever over short periods, as expected due to its smaller size and therefore lower $\beta$. Notably, at the longest averaging times, the force precision of the BioLever Mini shows worse performance than the long BioLever. This result is caused by the fixed amount of positional noise $(\delta x)$ in the 

$\delta x)$. The critical but non-intuitive result from this analysis is that the force precision of the longer cantilever starts to outperform the shorter cantilever at just $30 \mathrm{~ms}$ (Sullan et al., 2013). Hence, the standard view in AFM-based force spectroscopy that shorter cantilevers are better breaks down on a remarkably short timescale. Indeed, for standard gold-coated versions of these cantilevers, this crossover happens at a mere $10 \mathrm{~ms}$ (see also Fig. 6B) (Bull et al., 2014). Thus, choosing the optimum cantilever requires a careful consideration of the key requirements of a particular experiment and its duration.

\subsection{Temporal resolution in traditional force spectroscopy masks short-lived intermediates}

Long, soft cantilevers excel at force stability but are not universally better for force spectroscopy. They have increased force noise per unit bandwidth and relatively poor temporal precision. While the response time of an overdamped simple harmonic oscillator is well known $(\tau=\beta / k)$, our preferred metric is to measure the response time of the cantilever to an abrupt change in applied $F$. For traditional cantilevers, we measure the cantilever response by unfolding a small globular protein (Bull et al., 2014) because the transition path time for such proteins $(\sim 1-10 \mu \mathrm{s})$ (Chung et al., 2012; Lindorff-Larsen et al., 2011; Schuler and Hofmann, 2013) is much faster than a traditional cantilever can respond (50-1,000 $\mu \mathrm{s})$. Using this assay, the measured response of a long BioLever (Fig. 4A) was $450 \mu$ s (Fig. 4B) (Bull et al., 2014).

A recent study characterizing the folding and misfolding of the prion protein $(\mathrm{PrP})$ motivates the need for improved temporal resolution. In this work, Yu et al. (2012b) resolved the folded and unfolded states in an individual trajectory using a state-of-the-art optical trap (Fig. 4C). However, the short-lived misfolded states, labelled M1 and M2, were not well resolved in an individual trajectory. Rather, their existence was deduced as deviations away from the measured point-spread function of the instrument ( $\mathrm{Yu}$ et al., 2012b), which was possible because of the exceptional stability afforded by the dual-beam optical-trap geometry (Abbondanzieri et al., 2005; Greenleaf et al., 2005; Shaevitz et al., 2003). Such ultrastable optical-trapping instruments are not commercially available. Hence, there is an exciting scientific opportunity if the ease of use of a commercial AFM can be merged with significantly enhanced temporal resolution to directly detect such short-lived folding intermediates. 
Resolving briefly populated states is not the only reason to improve the temporal response of cantilevers. Recent theoretical work suggests proteins can unfold and refold on timescales faster than the force probe can respond (Nam and Makarov, 2016). Specifically, Nam and Makarov developed a simulation where the protein was connected to a bead held in an optical trap by a compliant handle (typically DNA in actual experiments). This simulation occasionally shows the protein (the molecular coordinate) folding and unfolding (i.e., hopping) between potential energy wells of the underlying energy landscape, while the slower-reacting bead shows no motion (Fig. $4 \mathrm{D}$, green star). Hence, the force probe acts as a low-pass filter. Although the simulation was carried out for the special case of an optical trap featuring a passive force clamp (Greenleaf et al., 2005), it acts as an important cautionary tale: the lack of temporal resolution can obscure the true molecular trajectory. A separate paper that used a combination of analytical analysis and simulation reached a similar conclusion: artifacts are introduced into SMFS results due to limited instrumental response time (Cossio et al., 2015). Thus, there is a clear need for SMFS probes with temporal resolution on the same timescale or faster than the time needed for the protein to cross the activation barrier ( 1-10 $\mu$ s) (Chung et al., 2012; Lindorff-Larsen et al., 2011; Schuler and Hofmann, 2013).

\subsection{Underdamped cantilevers are not optimal for force spectroscopy}

Continuing to decrease the size of cantilevers offers ever higher temporal resolution. Olympus BioLever Fasts (Fig. 4E) are commercially available ultrashort cantilevers that are only $9-\mu \mathrm{m}$ long and feature a characteristic frequency $\left(f_{0}\right)$ in liquid of $\sim 500 \mathrm{kHz}$. Such cantilevers were originally developed for high-speed AFM imaging (Ando, 2012) and are quite stiff by SMFS standards $(100 \mathrm{pN} / \mathrm{nm})$. The combination of high $k$ and low $\beta$ leads to a sub- $\mu$ s response time (Ando, 2012). Notwithstanding the challenges in detecting and calibrating such tiny cantilevers, Rico et al. (2013) had the insight to adapt a custom-built high-speed Ando-style AFM for SMFS. By pulling at velocities up to $4 \mathrm{~mm} / \mathrm{s}$, Rico et al. matched the pulling rate of a SMFS experiment with that of a molecular-dynamics (MD) simulation, facilitating the first direct comparison between steered-MD simulations and SMFS experiments.

Recently, we also mechanically unfolded a polyprotein with the same ultrashort cantilever on a commercial AFM (Cypher, Asylum Research) that featured a small spot-size laser $\left(9 \times 3 \mu \mathrm{m}^{2}\right)$ 
for detecting such tiny cantilevers. Our data yielded a $0.4-\mu$ s response time as the protein detached from the AFM tip (Fig. 4F) (Edwards et al., 2015), recapitulating the sub- $\mu$ s response time of previous experiments (Ando, 2012; Rico et al., 2013). After subtracting off the substantial optical-interference artifact common to ultrashort cantilevers, the resulting forceextension curves (Fig. 4G) show the classic saw-tooth pattern associated with unfolding a polyprotein (Rief et al., 1997). Hence, these fast-responding cantilevers can be used for SMFS on a commercial AFM, potentially improving throughput and ease-of-use. Moreover, such cantilevers have very low $\beta$, so they also offer improved force precision in addition to excellent temporal resolution.

Despite these compelling advantages, BioLever Fasts are also not optimal for SMFS. In particular, ultrashort cantilevers are not overdamped $(Q \leq 0.5)$, violating an underlying assumption that forms the basis of traditional force spectroscopy analyses (Bell, 1978; Dudko et al., 2006; Evans and Ritchie, 1997; Merkel et al., 1999). Traditional force spectroscopy analyses assume a uniform probability for unfolding over a sufficiently short period, whereas an underdamped cantilever $(Q>0.5)$ oscillates (Meirovitch, 1997). This oscillation modulates that unfolding probability at the resonance frequency of the cantilever $(\sim 500 \mathrm{kHz}$ for a BioLever Fast). To demonstrate that such oscillations happen while stretching a protein, we plot a small section of a force-vs-time trace while the cantilever was stretching a fully unfolded polyprotein. As shown in Fig. 4H, the force applied to the extended polyprotein undergoes periods of highfrequency force modulation at the resonance frequency of the cantilever. Moreover, the magnitude of these force fluctuations is large: $30-90 \mathrm{pN}$ peak-to-peak over a few $\mu \mathrm{s}$.

A simple back-of-the-envelope calculation is helpful to understand the potential impact of this observation. Within the Bell-Evans model (Evans and Ritchie, 1997), the unfolding rate varies as $\exp \left(F \Delta x^{\ddagger} / k_{\mathrm{B}} T\right)$, where the thermal energy $k_{\mathrm{B}} T$ equals $4.1 \mathrm{pN}-\mathrm{nm}$ and the distance to the transition state $\Delta x^{*}$ for NuG2 is $0.42 \mathrm{~nm}$ (He et al., 2015). Using an intermediate value for the force modulation of $60 \mathrm{pN}$, this calculation yields a $\sim 450$-fold variation in the unfolding rate during half an oscillatory cycle of the force probe. Besides complicating analysis, such rapid force modulation also obscures short-lived intermediates. Hence, improving temporal resolution and reducing $\beta$ are not the only factors that dictate an optimum force probe over short timescales. 
This calculation leads to an accompanying question: is such a cantilever-driven force variation applied to one end of a more compliant unfolded protein linker transmitted to the protein under study? Standard SFMS analysis assumes the force along the whole molecular construct is in equilibrium. Indeed, by modeling an unfolded protein using a worm-like-chain model, one assumes that the unfolded protein is fully exploring its conformational phase space more rapidly than the cantilever moves. The conformational rearrangement time for a small unfolded protein is $\sim 50-100 \mathrm{~ns}$, as measured by single-molecule fluorescence correlation spectroscopy (Soranno et al., 2012). While the longer polyprotein constructs used in AFM-based SMFS are expected to have a slower fundamental polymer relaxation mode (Doi and Edwards, 1986), the extended polyprotein is also under substantial tension and its conformational phase space is greatly restricted due to its extended end-to-end distance. Both these effects should substantially reduce the conformational reconfiguration time. Indeed, experimentally, the fundamental relaxation mode of an extended DNA molecule decreases with increasing extension (Quake et al., 1997). Hence, it is our expectation that the taut, unfolded protein explores its phase space more rapidly than the cantilever-driven oscillations and thus the protein under study is subject to the forces exerted by an oscillating cantilever. Further experimental and theoretical work is needed to confirm this expectation as well as probe the potential consequences of cantilever-driven oscillations on SMFS results.

\subsection{Choosing the right cantilever: balancing competing priorities}

Currently, there is no single best cantilever for all force spectroscopy measurements. In §2.1§2.4, we discuss how SMFS is limited by different aspects of the cantilever and illustrate how those limitations adversely affect SMFS experiments. Such knowledge is critical to choosing the proper cantilever for a particular experiment. Experiments that focus on measuring proteinfolding intermediates may need a cantilever with fast temporal resolution at the cost of force stability. In contrast, a force-clamp experiment measuring the lifetime of a folded protein at an elevated force would benefit from force stability at the cost of temporal resolution. In summary, by understanding the origin of these limitations and how they manifest in different cantilevers, users can make an informed choice for their particular application. Moreover, identifying these limitations enables researchers to develop techniques to overcome them. 


\section{Modifying commercial cantilevers for improved force spectroscopy}

Modern, small-format AFMs offer enhanced ease of use, throughput, and tip-sample stability. The primary limiting factor for AFM-based SMFS application-at least for our commercial $\mathrm{AFM}$ - is no longer the tip-sample stability but rather the cantilever and its detection. In this section, we discuss three methods to modify commercially available cantilevers to significantly increase their performance on a commercial AFM. In §3.1, we show that a simple 60-s chemical etch improves force stability 10 -fold to achieve sub-pN stability over $100 \mathrm{~s}$ in a biophysical assay. This sub-pN force precision was extended to span 5 decades of bandwidth along with improved temporal resolution and high reflectivity by modifying a shorter cantilever with an FIB (§3.2). Finally, in §3.3, FIB modification was extended to ultrashort BioLever Fast cantilevers to optimize them for SMFS rather than tapping-mode imaging. To detect the modified ultrashort cantilevers, we retrofitted our commercial AFM with a home-built detection module featuring a $3-\mu \mathrm{m}$ circular spot size. While this fabrication process is significantly more involved, these cantilevers, along with the instrumental improvements to detect them, led to significantly improved force precision over unmodified ultrashort cantilevers while retaining a $1-\mu$ s temporal resolution.

\subsection{Sub-pN stability over 100 s: simple and repeatable}

As stated in $§ 2$, significant drift in the measured force occurred while holding a biomolecule under constant extension with our ultrastable AFM. Moreover, a similar level of drift in $z_{0}$ was observed in a modern commercial AFM (Fig. 3A orange; 5A, gold). A simple experiment on the commercial AFM isolated the cantilever as the primary source of that drift. Specifically, we positioned the detection laser on the chip at the base of the cantilever and measured an 800-fold reduction in the measured force drift over 5 min (Churnside et al., 2012). Hence, this force drift does not originate from other opto-mechanical sources within the detection system (e.g., pointing and mode noise in the detection laser or motion of the quadrant photodiode). Rather, motion in $z_{0}$ is the primary source, even hours after immersing the cantilever in liquid. A pair of short ( $\sim 30 \mathrm{~s})$ chemical etches removes the gold and underlying chromium coatings on the long BioLever and thereby dramatically decreased the observed drift (Fig. 5A, red). 
The adverse effect of the gold coating on a cantilever's stability affected multiple types of cantilevers (Sullan et al., 2013). The magnitude of the effect for single-side and fully coated cantilevers was similar, on a temperature stabilized AFM. Hence, the bi-metallic effect, which makes single-side-coated cantilevers very sensitive to temperature changes (Radmacher et al., 1995), is not the primary source of the problem. We speculate that the origin of the problem is stress introduced into the gold coating during the process of wetting the cantilever. Specifically, as the cantilever goes through the air-water interface, it often folds up against the chip that supports the cantilever (Fig. 5B) and then unfolds after a variable period of time. Two observations support this speculation. First, we directly observed damage in the gold coating after unfolding (Fig. 5C) (Churnside et al., 2012). Second, the direction of drift has a strong bias, rather than being randomly distributed (Churnside et al., 2012). Hence, we do not think the origin of this drift is simply due to the viscoelasticity of the gold coating previously reported in high-resolution studies of gold-coated cantilevers in air (Paolino and Bellon, 2009). Future work that conclusively demonstrated the origin of this unwanted effect could allow manufacturers to develop better reflective coatings that minimize metallization-induced drift.

The key experimental question to address for uncoated cantilevers is: does the accompanying 5-10-fold reduction in sensitivity adversely affect one's ability to measure the position of the cantilever? Traditionally, it is assumed that high sensitivity $(\mathrm{mV} / \mathrm{nm})$ and therefore high reflectivity is critical to making precision measurements with an AFM. To test this assumption, we computed the positional PSD for these soft $(6 \mathrm{pN} / \mathrm{nm})$ long BioLevers (Fig. 5D). The resulting data show that the cantilever's high-frequency thermal motion was equally well resolved for both the coated and uncoated cantilever. Hence, there was no loss in the positional precision to detect such a soft, uncoated cantilever up to its characteristic frequency $\left(f_{0} \approx 2 \mathrm{kHz}\right)$. Importantly, this analysis also shows that an uncoated cantilever exhibits a $\sim 100$-fold reduction in instrumental noise at $0.01 \mathrm{~Hz}$ compared to a gold-coated cantilever. More generally, we showed that sub-pN stability over $100 \mathrm{~s}$ is now routinely achievable on a commercial AFM just 30 min after putting the cantilever into liquid (Churnside et al., 2012).

Researchers are already achieving new results in high-precision AFM-based SMFS studies of proteins using uncoated long BioLevers. While pioneering AFM studies have previously probed the near-equilibrium folding of fully $\alpha$-helical proteins (Junker et al., 2009; Lee et al., 2010), 
such near-equilibrium studies of proteins that contain fully $\beta$-sheet structures or a mixture of $\alpha / \beta$ structures were previously inaccessible due to their slow refolding. By using highly stable uncoated cantilevers, He et al. (2015) measured the near-equilibrium folding and unfolding of $\mathrm{NuG} 2$, an $\alpha / \beta$ protein, on a commercial AFM at pulling speeds of $2 \mathrm{~nm} / \mathrm{s}$ (Fig. $5 \mathrm{E}$ ). At such low speeds, repeated folding and unfolding is seen in both the retraction and subsequent approach curves, opening the door to these exciting studies over a broader range of proteins.

\subsection{Focused-ion-beam modified cantilevers: sub-pN precision over 5 decades of bandwidth}

While uncoated long BioLevers are an accessible means to sub-pN bioAFM, an ongoing goal in protein-folding studies is detecting small, short-lived intermediates (Cecconi et al., 2005; Sapra et al., 2006; Stigler et al., 2011; Yu et al., 2012b). Such detection requires improved force precision and temporal resolution. The path towards improved force precision is well established: reduce the hydrodynamic drag $(\beta)$ of the cantilever, as previously discussed (\$2.2) (Viani et al., 1999). Unfortunately, the long soft cantilevers that provide the best force stability suffer from relatively poor temporal resolution (450 $\mu$ s; see Fig. 4B) due to their increased $\beta$ and decreased $k(\tau=\beta / k)$. This reduced temporal resolution, coupled with decreased short-term force precision, hinders their application in SMFS studies. Furthermore, removing the gold coating also leads to a 10-fold decrease in sensitivity, which can be essential in applications at higher frequencies and those that use stiffer cantilevers. Hence, highly reflective cantilevers that are soft and sensitive, while still being responsive, are needed.

To meet the above requirements, the optimum cantilever needs reduced $\beta$ for short-term force precision and reduced $k$ for long-term stability. However, shorter cantilevers are inherently stiffer $\left(k \sim 1 / L^{3}\right)$. We circumvented this scaling relation by using an FIB to modify a short cantilever ( $L$ $=40 \mu \mathrm{m}$; BioLever Mini) (Fig. 6A) (Bull et al., 2014). Our efficient process, building on earlier work (Hodges et al., 2001; Maali et al., 2006), led to a $\sim 10$-fold reduction in both $k$ and $\beta$ near the surface. Further, we preserved the benefits of a highly reflective cantilever while also mitigating the adverse effects of a gold coating on force stability. Specifically, we introduced a small transparent capping layer that preserved a patch of gold at the end of the cantilever during the subsequent chemical etches. Collectively, this set of cantilever modifications extended AFM's sub-pN bandwidth by a factor of $\sim 50$ to span five decades of bandwidth ( $\Delta f \approx 0.01-1,000$ 

gold), these FIB-modified BioLever Minis had improved short-term force precision due to their reduced $\beta$ and dramatically improved force stability due to their reduced $k$ and spatially localized gold coating.

In the context of protein-unfolding assays, FIB-modified BioLever Minis showed improved short-term force precision coupled with state-of-the-art force stability by AFM standards (Fig. 6C-D). Moreover, because both $\beta$ and $k$ were reduced 10-fold, their temporal resolution remained excellent $(\sim 75 \mu \mathrm{s})$ (Fig. $2 \mathrm{~B})$, similar to an unmodified BioLever Mini. Unlike the unmodified BioLever Minis, the modified cantilevers were overdamped $(Q=0.33)$ fulfilling an underlying assumption for most SMFS analysis (Bell, 1978; Dudko et al., 2006; Evans and Ritchie, 1997; Merkel et al., 1999). Modified cantilevers were also robust and reused for SMFS over multiple days.

The barrier to making your own FIB-modified cantilevers may seem unreasonably high. However, we note that our lab started this FIB-modification project with no previous experience in using an FIB. Moreover, while we tested many different fabrication protocols, the current version is reproducible across multiple individuals and has been essentially unchanged over the last year. Indeed, an undergraduate in our lab developed this process and a current undergraduate can modify 2-4 cantilevers per hour of FIB time (Bull et al., 2014). Thus, a robust protocol is available. For a new lab member, it takes $\sim 10-20 \mathrm{~h}$ of one-on-one training, if the new individual has modest experience with SEM or FIB. Those with no prior training would need to be trained in FIB use, which can range from 4-24 h depending on individual FIB facilities. In the absence of direct one-on-one training, we estimate an experienced FIB/SEM user could become proficient in $\sim 10-20 \mathrm{~h}$, and non-experts in $\sim 50-100 \mathrm{~h}$.

In summary, FIB-modified BioLever Minis are an excellent all-purpose cantilever for SMFS. They offer significantly improved performance over commercially available cantilevers while also maintaining high reflectivity (Fig. 6B). These advantages are not limited to SMFS but can also be applied to other areas of AFM use in biology, such as the sub-nanometer nanomechanical mapping of membrane proteins (Medalsy et al., 2011; Rico et al., 2011). The most significant barriers to making them are $(i)$ access to an FIB, and (ii) the upfront investment in time and 
cantilevers to become trained in fabricating them. In the long run, we anticipate that commercial cantilever manufacturers will adopt the strategies outlined here to make such cantilevers more accessible to the broader community. Indeed, this process has started; there is now a commercially available cantilever with a small gold patch at the end of the cantilever that exhibits reduced force drift (Uniqprobe, Nanosensors).

\subsection{Ultrashort cantilevers optimized for SMFS: 1 - $\mu$ s resolution on a commercial AFM.}

In SMFS, AFM offers a unique capability over optical traps and magnetic tweezers: sub- $\mu$ s temporal resolution in an individual record when using an ultrashort cantilever (Rico et al., 2013). Although commercially available ultrashort cantilevers offer both improved temporal resolution and force precision, they are designed for tapping-mode imaging rather than SMFS (Ando, 2012). In particular, these cantilevers are underdamped $(Q \approx 1)$, causing a high-frequency force modulation not accounted for in traditional force spectroscopy analyses (Fig. 4H). To optimize ultrashort cantilevers for SMFS, we adapted our FIB-modification protocol to these cantilevers (Fig. 7A) (Edwards et al., 2015). The main challenge in the fabrication of such cantilevers is that FIB-induced cutting leads to significant upward bending. Fortuitously, thinning the cantilever's remaining narrow supports straightens out the cantilever and yields a typical final stiffness of $20-40 \mathrm{pN} / \mathrm{nm}$. While we had to invest significantly in establishing this process, a skilled undergraduate currently makes $2-4$ cantilevers in $1 \mathrm{~h}$ of FIB time.

The next step was to merge such soft, but fast responding, cantilevers with the ease of use and throughput of a commercial AFM. Unfortunately, the resulting sensitivity was too low to yield interpretable data, even when using a commercial AFM that featured a specialized small spotsize module $\left(9 \times 3 \mu^{2}\right)$ designed to detect an unmodified BioLever Fast. To overcome this problem, we developed a homemade-detection module that has a 3- $\mu \mathrm{m}$ circular spot size (Edwards et al., 2015). Importantly, this module could be swapped in and out of our commercial AFM in $\sim 15$ min, so that its general utility was not sacrificed.

After this hardware upgrade, we could quantitatively compare a modified BioLever Fast (Fig. 7B, blue) to three other cantilevers recently used to advance AFM-based SMFS: an uncoated long BioLever (Fig. 7B, red) (Churnside et al., 2012), an FIB-modified BioLever Mini (Fig. 7B, 
green) (Bull et al., 2014), and an unmodified BioLever Fast (Fig. 7B, purple) (Rico et al., 2013). To do so, we computed the force PSD of the thermal motion of the cantilever when the cantilever was $50 \mathrm{~nm}$ over the surface, a typical height of an AFM-based SMFS assay. This analysis accurately accounts for the impact of squeezed film damping (i.e., increased $\beta$ ) when the cantilever is near a surface. The resulting comparison shows that both the modified and unmodified BioLever Fasts had better force precision in the thermally limited regime and dramatically higher characteristic frequencies $\left(f_{0}\right)$. As expected, the modified BioLever Fast exhibited a lower $f_{0}$, but the reduction was only 2 -fold for a 3 -fold reduction in $k$. On the other hand, the softer long BioLever $(k=4.3 \mathrm{pN} / \mathrm{nm})$ and modified BioLever Mini $(k=4.7 \mathrm{pN} / \mathrm{nm})$ exhibited better performance at low frequencies due to positional noise $(\delta x)$ in the optical lever arm (Fig. 3D, black). For the same reason, the softer modified BioLever Fast ( $k=40 \mathrm{pN} / \mathrm{nm}$ ) showed better force stability than the stiffer unmodified BioLever Fast $(k=130 \mathrm{pN} / \mathrm{nm})$. Importantly, analysis of these PSDs showed that the modified BioLever Fast has a reduced $Q$ in comparison to the unmodified BioLever Fast ( $Q=0.52$ vs $Q=0.85$, respectively), which reduces the high-frequency force modulation from underdamped motion.

To compare the biophysical performance of FIB-modified ultrashort cantilevers to unmodified ones, we mechanically stretched a polyprotein of $\mathrm{NuG} 2$ with three different ultrashort cantilevers: a standard BioLever Fast (Fig. 7C, purple) and two modified BioLever Fasts of different stiffnesses $[k=40 \mathrm{pN} / \mathrm{nm}$ (Fig. 7C, blue) and $20 \mathrm{pN} / \mathrm{nm}$ (Fig. 7C, cyan)]). For all three cantilevers, the resulting force-extension traces exhibited the classic saw-tooth pattern (Rief et al., 1997), after computationally subtracting off a substantial optical-interference artifact (not shown). Analysis of these curves with the worm-like-chain model yielded an increase in contour length in quantitative agreement with prior studies (Bull et al., 2014; Cao et al., 2007; He et al., 2015). Significantly, modifying the cantilevers led to substantially improved force precision in high-bandwidth data $(5 \mathrm{MHz})$ while stretching an unfolded polyprotein (Fig. 7D). This improvement arises from both the softer cantilever acting as a low-pass filter and from the reduction in $Q$ that minimizes cantilever ringing. Perhaps most importantly, despite the reduction of $k, f_{0}$, and $Q$, the modified BioLever Fasts maintained a 1- $\mu$ s-scale response time as measured by the force decay following detachment of the protein from the tip (Fig. 7E). An additional advantage of FIB modification is that the cantilever's mechanical properties can be tuned for a 
particular experiment. Increased force precision $(6.8 \mathrm{pN}$ vs $11 \mathrm{pN}, \mathrm{RMS}$ at $5 \mathrm{MHz})$ is traded for a modest reduction in response time (1.8 $\mu \mathrm{s}$ vs $1.2 \mu \mathrm{s})$ when using a slightly softer cantilever (20 $\mathrm{pN} / \mathrm{nm}$ vs $40 \mathrm{pN} / \mathrm{nm}$ ). Summarizing, these cantilevers should excel at detecting folding intermediates with lifetimes short compared to the typical time resolution of SMFS (50-1,000 $\mu \mathrm{s})$. More generally, modified ultrashort cantilevers are an exciting avenue for exploring highprecision SMFS measurements with $1-\mu$ s resolution in a manner consistent with traditional SMFS analysis.

However, these performance gains come at an increased cost. The most significant hurdle to implementation for most users will be achieving a sufficiently small laser spot size for detecting the modified BioLever Fast. On our AFM (Cypher, Asylum Research), we developed a detection module that was almost entirely external to the AFM (Edwards et al., 2015). The new detection module was fiber-coupled into the AFM using an existing mounting interface for different detection modules, so none of the other optics in the AFM detection path were modified. The total construction time for this laser module (including the associated optics and electronics for intensity stabilization) is $1-3$ months, depending on the user's expertise. We also note that ultrashort cantilevers in general are harder to calibrate. We followed the protocol developed for the original SMFS study with ultrashort cantilevers (Rico et al., 2013), where $k$ is calibrated in air (Sader et al., 1999; Sader et al., 2012) and sensitivity is deduced by analyzing the PSD in liquid (Higgins et al., 2006). This two-step procedure could be simplified by reducing the optical-interference artifact embedded in the deflection signal. This optical-interference artifact complicates calibration, data collection, and data analysis. While computational post-processing can nearly eliminate this effect, small $(\sim 1-5 \mathrm{pN})$ residual manifestations generally remain. Further, this interference artifact significantly limits more sophisticated real-time event-driven data collection protocols since the magnitude of the biological signal is typically smaller than the interference artifact for ultrashort cantilevers. Finally, while ultrashort cantilevers are more challenging to reliably FIB modify, they can be modified more rapidly than a BioLever Mini due to their decreased size. For a user familiar with the procedure of making a modified BioLever Mini, we estimate 5-10 h to become proficient at modifying a BioLever Fast.

Despite these hurdles, the exceptional temporal resolution of these cantilevers and their improved force precision should enable enhanced characterization of protein folding. In turn, 
new scientific insights derived with such modified ultrashort cantilevers can be expected to spur further developments in cantilever technology and hopefully wafer-scale implementation of cantilever modifications that will allow for broader adoption.

\section{Conclusions and future direction}

More than two decades after the start of AFM-based SMFS (Florin et al., 1994; Lee et al., 1994a; Lee et al., 1994b; Moy et al., 1994), innovation continues. Over the last few years, our group has identified a number of areas in which standard commercially available cantilevers limit such applications. In $\S 2$, we enumerate several of these limitations and how they adversely affect SMFS. In $\S 3$, we show that modifying cantilevers mitigates these problems and demonstrate the resulting benefits to SMFS. Most importantly, we hope that researchers make an informed choice on the cantilever they use. As discussed in this article, choosing the appropriate cantilever for a particular application involves a tradeoff. One can have a stiff, low-force noise cantilever with relatively poor long-term stability. Alternatively, one can get excellent long-term force stability, but with increased force noise per unit bandwidth and decreased temporal resolution. With some investment in both time and effort, one can dramatically improve data quality by making an FIBmodified BioLever Mini. This cantilever is, perhaps, the best general purpose AFM cantilever for SMFS, achieving sub-pN force precision over 5 decades of bandwidth while still retaining high reflectivity.

For more specialized applications, an FIB-modified BioLever Fast offers 1- $\mu$ s temporal precision in a manner consistent with traditional SMFS theories. Additionally, such cantilevers achieve a short-term force precision at $200 \mu$ s that is just $50 \%$ more than state-of-the-art optical trapping instruments (Yu et al., 2012b). While the modification of such tiny cantilevers takes less time than the larger BioLever Mini, one also needs to invest in modifying a commercial AFM to detect them. The home-built detection module that we developed is inherently specific to our particular AFM. For those willing to make this investment, there are a several additional advantages. Specifically, this $3-\mu \mathrm{m}$ circular-spot-size detection laser offers better sensitivity and reduced optical-interference artifacts compared to the commercially available small spot-size module, when using the unmodified BioLever Fasts and the larger modified BioLever Minis. 
With these recent performance gains in AFM-based SMFS, it is useful to compare AFM to optical traps and magnetic tweezers. Over the last decade, optical traps have become the premiere instrument for studying the equilibrium folding and unfolding of proteins and nucleic acid structures (Cecconi et al., 2005; Liphardt et al., 2001; Stigler et al., 2011; Woodside et al., 2006b; Yu et al., 2012a). The key advantage of optical traps is their force stability and force precision (Greenleaf et al., 2007; Neuman and Nagy, 2008). However, advanced dual-beam optical traps are custom-built instruments that require significant expertise to build and operate. Additionally, optical-trapping-based assays generally use relatively long, compliant handles $(\sim 300-1,000 \mathrm{~nm})$ relative to AFM $(\sim 30-100 \mathrm{~nm})$. Magnetic tweezers offer excellent force stability in conjunction with the ability to simultaneously measure hundreds to thousands of molecules in parallel (De Vlaminck et al., 2011). Magnetic tweezer, however, suffer from relatively poor temporal resolution $(\sim 0.01-0.1 \mathrm{~s})$ in comparison to standard optical-trapping and AFM assays (50-1,000 $\mu \mathrm{s})$ (Greenleaf et al., 2007; Neuman and Nagy, 2008), which themselves may not have enough temporal resolution to accurately capture the true molecular trajectory (Fig. 4D) (Nam and Makarov, 2016). Hence, the availability of highly stable, easy-to-use commercial AFMs offers an exciting opportunity to broaden the range of users and systems studied by highprecision SMFS.

To realize AFM-based SMFS's full potential, there are still a number of hurdles to increasing data quality and data quantity. Smaller cantilevers will continue to offer improved force precision and temporal resolution. Indeed, the Ando group, in collaboration with Olympus, is already applying next-generation ultrashort cantilevers that are just 7- $\mu \mathrm{m}$ long (Ando et al., 2014) (although these cantilevers are not yet commercially available). To fully exploit the increased force precision and temporal resolution of such short cantilevers, their detection needs to be thermally limited over longer times, which necessitates addressing the instrumentational noise in the optical lever that dominates their low-frequency detection (Fig. 3D, black). Such tiny cantilevers also exhibit a significant optical-inference artifact and remain technically challenging to calibrate, decreasing throughput and broader accessibility. A parallel path to improving data quality and data quantity focuses on site-specific coupling of biomolecules to surfaces and AFM tips. Innovative solutions are being developed by a large number of groups (Otten et al., 2014; Popa et al., 2013; Stahl et al., 2012; Taniguchi and Kawakami, 2010; Zakeri et al., 2012; 
Zimmermann et al., 2010), although the published protocols still require significant daily investments in time and labor.

Overall, we are very optimistic about AFM's future role in SMFS. The combination of modified ultrashort cantilevers along with the instrumental improvements to detect them on a commercial AFM opens the door to probing protein folding dynamics with an unprecedented combination of force precision and temporal resolution. Continued methodological improvements should expand the duration of such high-precision studies and the ease with which they are applied to a diverse array of biological systems. Finally, AFM has a unique ability to image and manipulate biological structures with sub-nm precision. While we have discussed these improvements in the context of AFM-based SMFS, AFM's application to biomedical research is much larger than SMFS (Muller and Dufrene, 2008). Hence, we expect that many other exciting biophysical applications of AFM can immediately benefit from the improvements discussed here.

\section{Acknowledgements:}

The authors thank Dima Makarov, Michael Woodside, and Hao Yu for sharing data. This work was supported by a fellowship from the National Research Council (D.T.E.), the National Science Foundation (DBI-135398; Phys-1125844), and NIST. Mention of commercial products is for information only; it does not imply NIST's recommendation or endorsement. T.T.P. is a staff member of NIST's Quantum Physics Division. 

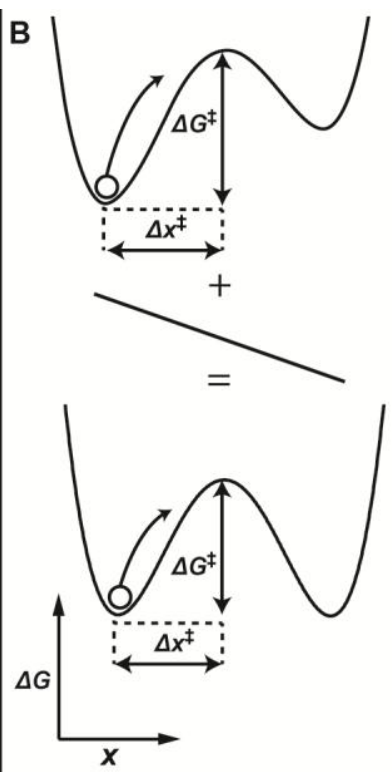

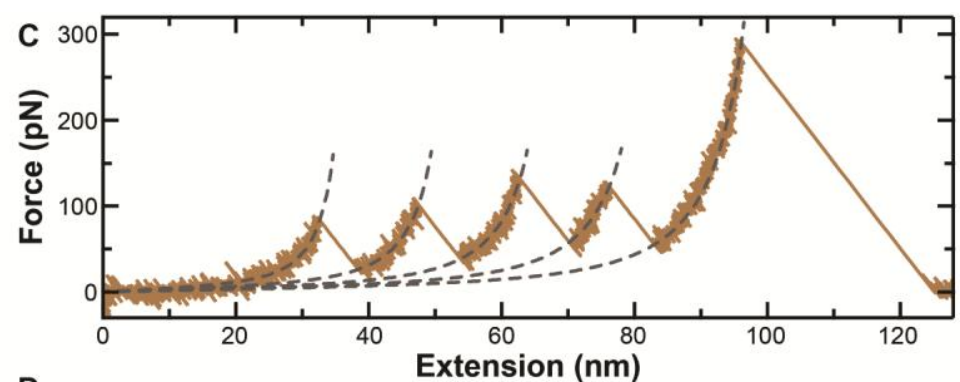

D

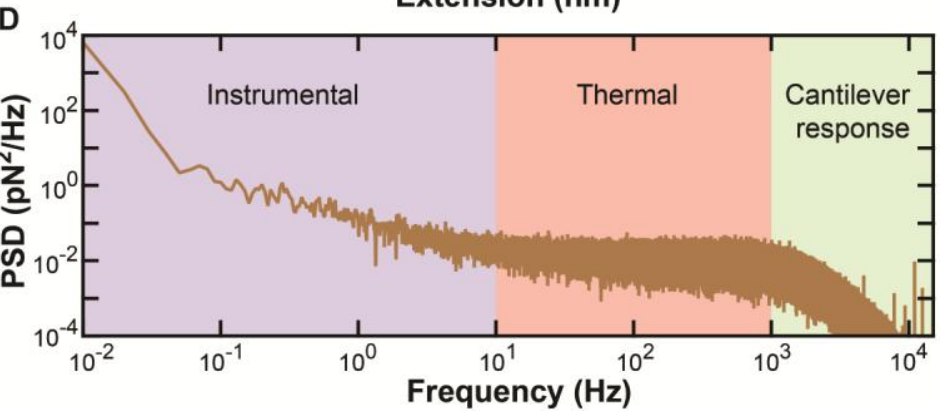

Fig. 1. Single-molecule force spectroscopy of a polyprotein. (A) A cartoon of the assay showing a polyprotein composed of four domains of $\mathrm{NuG} 2$ being mechanically stretched by a gold-coated long BioLever. (B) In the simplest description of the force-assisted unfolding process, the application of a constant force $(F)$ lowers the height of the energy barrier $\left(\Delta G^{*}\right)$ at the transition state $\left(\Delta x^{\ddagger}\right)$ by $F \Delta x^{\ddagger}$, effectively "tilting" the folding energy landscape. (C) Force-extension record showing the mechanical unfolding of the NuG2 polyprotein as the gold-coated cantilever $(k=10.1 \mathrm{pN} / \mathrm{nm})$ is retracted at $400 \mathrm{~nm} / \mathrm{s}$. Data recorded at $50 \mathrm{kHz}$. Grey dashed lines represent worm-like-chain fits to the data, yielding a change in contour length $\left(\Delta L_{0}\right)$ of $16.2-17.0 \mathrm{~nm}$, consistent with previous results (Bull et al., 2014; Cao et al., 2007; He et al., 2015). (D) Averaged force power spectral density (PSD) of five consecutive 100-s records of the zero-force position $\left(z_{0}\right)$ for a gold-coated long $\operatorname{BioLever}(k=6.8 \mathrm{pN} / \mathrm{nm})$ after equilibrating in liquid for $>2$ h. Three different regimes exist, one dominated by instrumental noise (purple), one limited by thermal motion of the cantilever (peach), and one determined by the mechanical response time of the cantilever (green). 
A Uncoated long Biolever
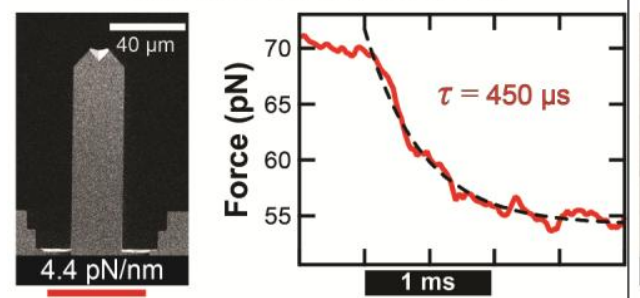

Pros: - Excellent force stability

-Easy to prepare

Cons: $\cdot$ Moderate temporal resolution

- Moderate force precision

- Low reflectivity
B FIB-modified Biolever Mini
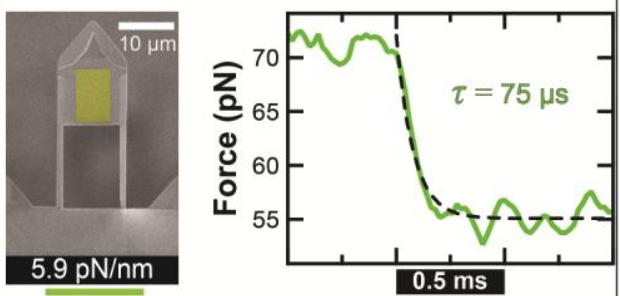

Pros: - Excellent force stability

- Good force precision

- Good temporal resolution

-High reflectivity

Cons: • Requires FIB modification
C FIB-modified Biolever Fast
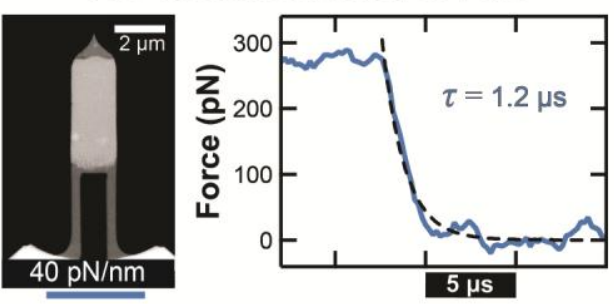

Pros: - Excellent temporal resolution

- Excellent force precision

-Avoids cantilever ringing

Cons: • Requires FIB modification

- Requires custom detection laser

- Moderate stability

Fig. 2. Improving force spectroscopy with three modified, commercial cantilevers. (A) An uncoated long BioLever, (B) a focused-ion-beam (FIB)-modified BioLever Mini, and (C) an FIB-modified BioLever Fast. Scanning electron microscopy (SEM) images of each cantilever and its stiffness are shown at the top left of each column. A force-versus-time record detailing the response time of each cantilever to an abrupt change in $F$ is shown at the top right of each column. Time constants were determined by an exponential fit (dashed line) to each record. For the uncoated long BioLever and FIB-modified BioLever Mini, the traces shown are the averaged step-response function during protein unfolding at $50 \mathrm{kHz}$. For the FIB-modified BioLever Fast, the record shown is the response to detachment of the polyprotein from the tip recorded at 5 MHz. SEM images in A and B are reprinted with permission from (Bull et al., 2014). The SEM image in $\mathrm{C}$ is reprinted with permission from (Edwards et al., 2015). 

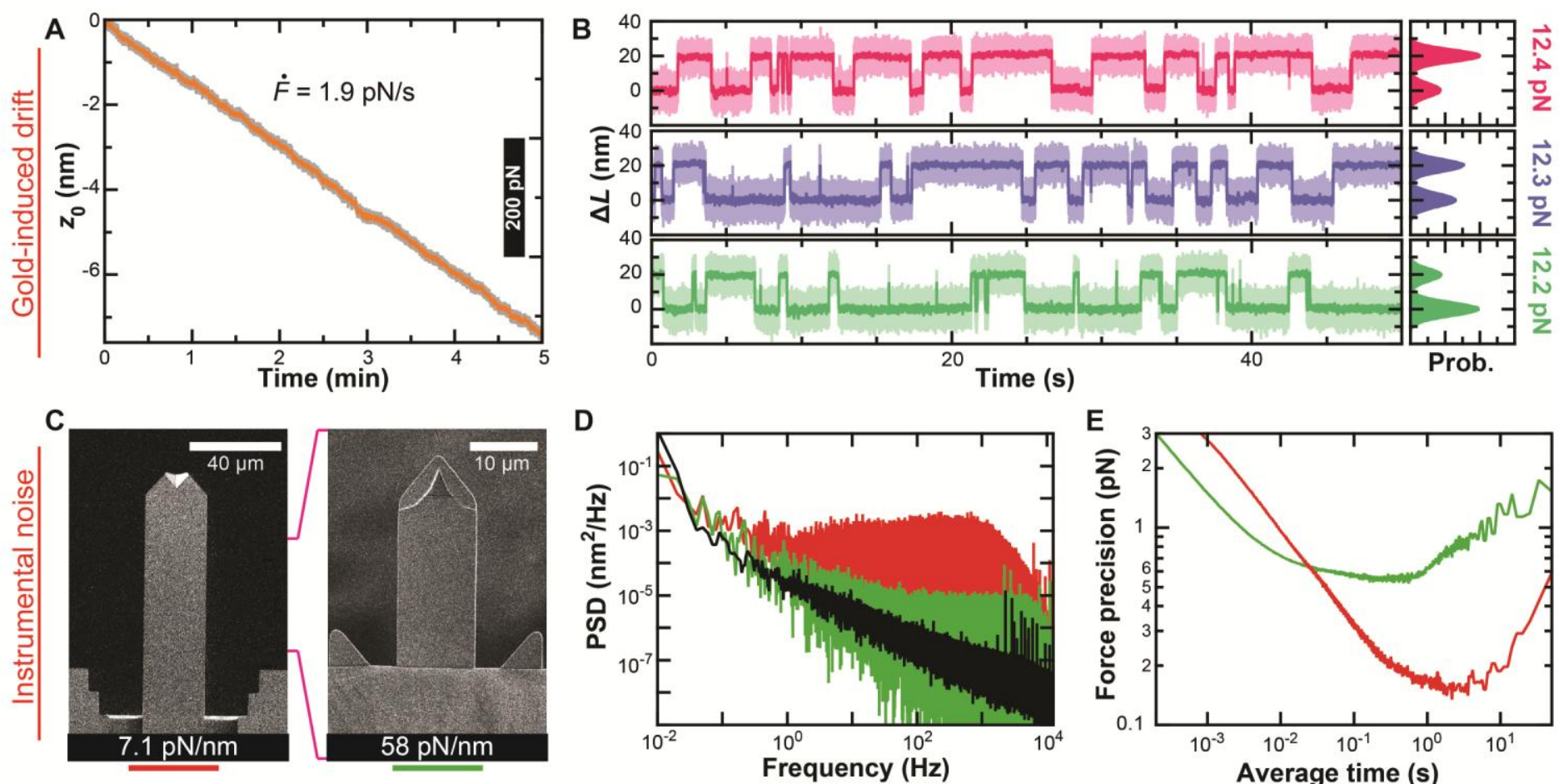

Fig. 3. Limitations in force stability and precision using commercial cantilevers. (A) A 5-min trace shows the zero-force position $\left(z_{0}\right)$ of a gold-coated BioLever Mini cantilever $(k=72$ $\mathrm{pN} / \mathrm{nm}$ ) at $\sim 2 \mathrm{~h}$ after loading into liquid. Data collected at $5 \mathrm{kHz}$ (grey) and filtered to $50 \mathrm{~Hz}$ (orange). (B) Extension-vs-time traces showing a DNA hairpin opening and closing in an optical trap under a constant load of $12.4 \mathrm{pN}, 12.3 \mathrm{pN}$, and $12.2 \mathrm{pN}$ (top, middle, and bottom panels, respectively). Data filtered to $2 \mathrm{kHz}$ (shaded) and $100 \mathrm{~Hz}$ (solid). Histograms of the data show that the probability of being unfolded increases from $29 \%(12.2 \mathrm{pN})$ to $52 \%(12.3 \mathrm{pN})$ and finally to $68 \%(12.4 \mathrm{pN}$ ). Adapted from (Carter et al., 2009). (C) SEM images of an uncoated long BioLever $(L=100 \mu \mathrm{m})$ and an uncoated BioLever Mini $(L=40 \mu \mathrm{m})$. Each cantilever's measured spring constant is indicated. The color of the line below each image is associated with curves in D-E. Reprinted with permission from (Bull et al., 2014). (D) Positional PSDs of an uncoated long BioLever and BioLever Mini cantilever calculated from 100-s traces at $\sim 2 \mathrm{~h}$ after loading into liquid. At low frequencies, these two traces are limited by the optical-lever-arm detection limit (black), deduced by reflecting the laser off of the chip holding the cantilever. (E) Average force precision, technically the Allan deviation (Sullivan et al., 1990), as a function of averaging time for an uncoated long BioLever and an uncoated BioLever Mini using the data from D. Initially, the force precision improves with increased averaging until instrumental limitations lead to degraded performance over longer times. Adapted from (Sullan et al., 2013). 

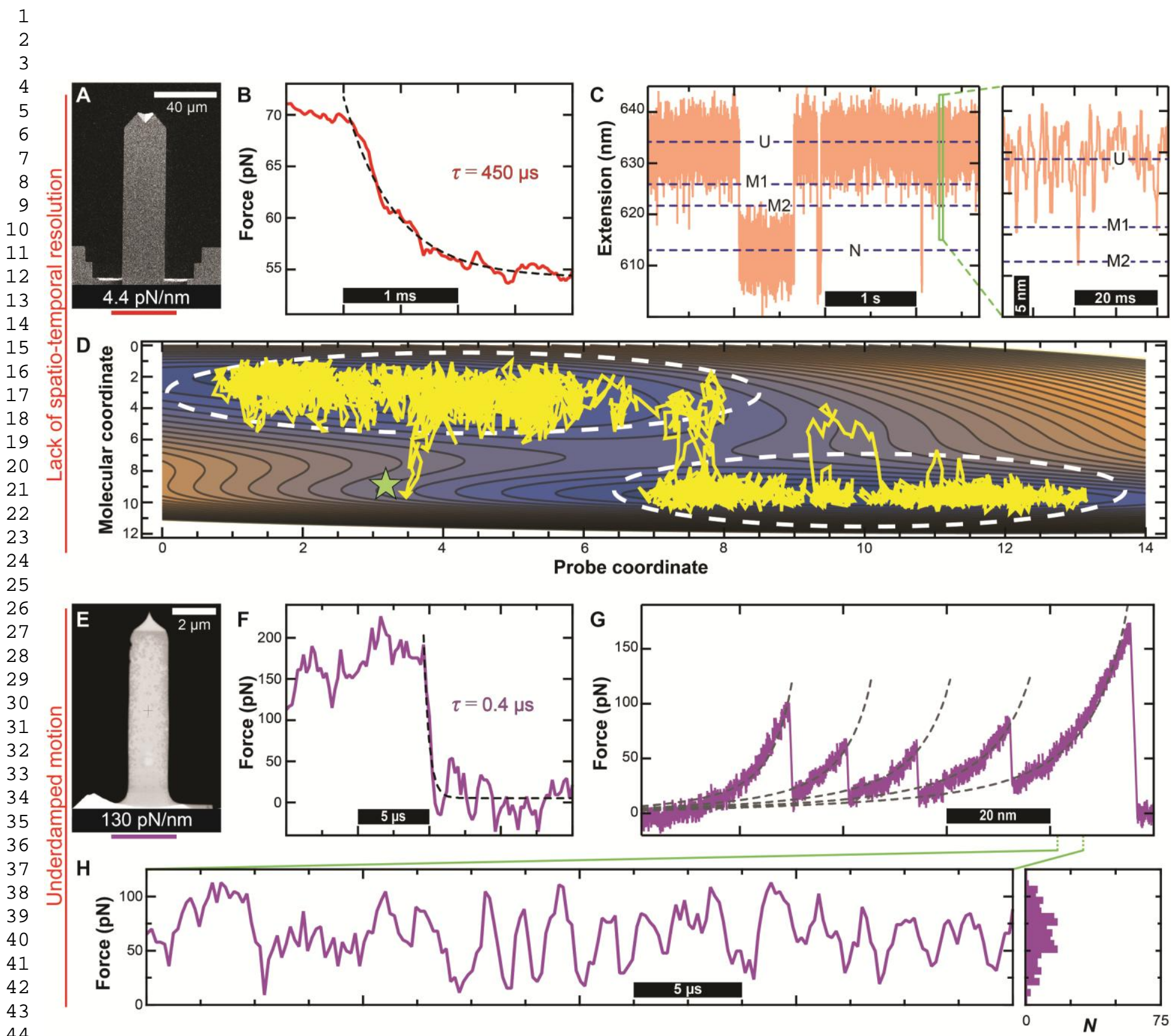

Fig. 4. Limitations to SMFS due to AFM cantilever response in liquid. (A) SEM image of an uncoated long BioLever $(L=100 \mu \mathrm{m})$. The cantilever's measured spring constant is indicated. The color of the line below the image is associated with the curve in B. Reprinted with permission from (Bull et al., 2014). (B) Averaged force-versus-time record shows the stepresponse function of a long BioLever after a protein domain unfolds. The characteristic time for this cantilever was determined by a fit to a single exponential (dashed line). Adapted from (Bull et al., 2014). (C) Extension-versus-time record showing the folding and unfolding of the prion 

M2) are observed. The inset shows an expanded region. Adapted from (Yu et al., 2012b). (D) Simulated experimental trajectory showing the folding and unfolding of a macromolecule (molecular coordinate) and the response of an optically trapped bead (probe coordinate) in a twodimensional energy landscape (background) (Nam and Makarov, 2016). The majority of the trajectory (yellow) shows the bead coordinate properly tracking the molecular coordinate in one of two states in the top left and lower right quadrants (white dashed ovals). However, the molecule occasional folds and unfolds by moving across the energy barrier (along the $y$-axis) without a corresponding change in the bead position (star), implying a lack of temporal resolution to fully resolve molecular motion. This simulation contains a macromolecule, compliant linkers between the macromolecule, and a bead trapped in a passive optical trap. It was carried out under the conditions that the linkers were soft compared to the barrier curvature $(0.5 \mathrm{pN} / \mathrm{nm}$ vs $3.23 \mathrm{pN} / \mathrm{nm})$ and that the drag on the bead is 100 -fold greater than the molecular friction. Adapted from (Nam and Makarov, 2016). (E) SEM image of a gold-coated BioLever Fast $(L=9 \mu \mathrm{m})$. The cantilever's measured spring constant is indicated. The color of the line below the image is associated with curves in $\mathrm{F}-\mathrm{H}$. Reprinted with permission from (Edwards et al., 2015). (F) Force-versus-time record showing the response of BioLever Fast cantilever as the protein detaches from the tip. Data recorded at $5 \mathrm{MHz}$. Time constant determined by an exponential fit (dashed line). (G) Force-extension record showing the mechanical unfolding of an $\mathrm{NuG} 2$ polyprotein at $400 \mathrm{~nm} / \mathrm{s}$ with a standard BioLever Fast after subtraction of an opticalinterference artifact. Data recorded at $50 \mathrm{kHz}$. Grey dashed lines represent worm-like-chain fits to the data, yielding a change in contour length $\left(\Delta L_{0}\right)$ of $17.9 \mathrm{~nm}$, in agreement with previous results (Bull et al., 2014; Cao et al., 2007; He et al., 2015). (H) Force-versus-time record as a fully unfolded polyprotein is stretched at $\sim 60 \mathrm{pN}$. A histogram shows the distribution in measured forces after a linear subtraction, with an RMS deviation of $24 \mathrm{pN}$. Data recorded at 5 MHz. Panels F-H adapted from (Edwards et al., 2015). 

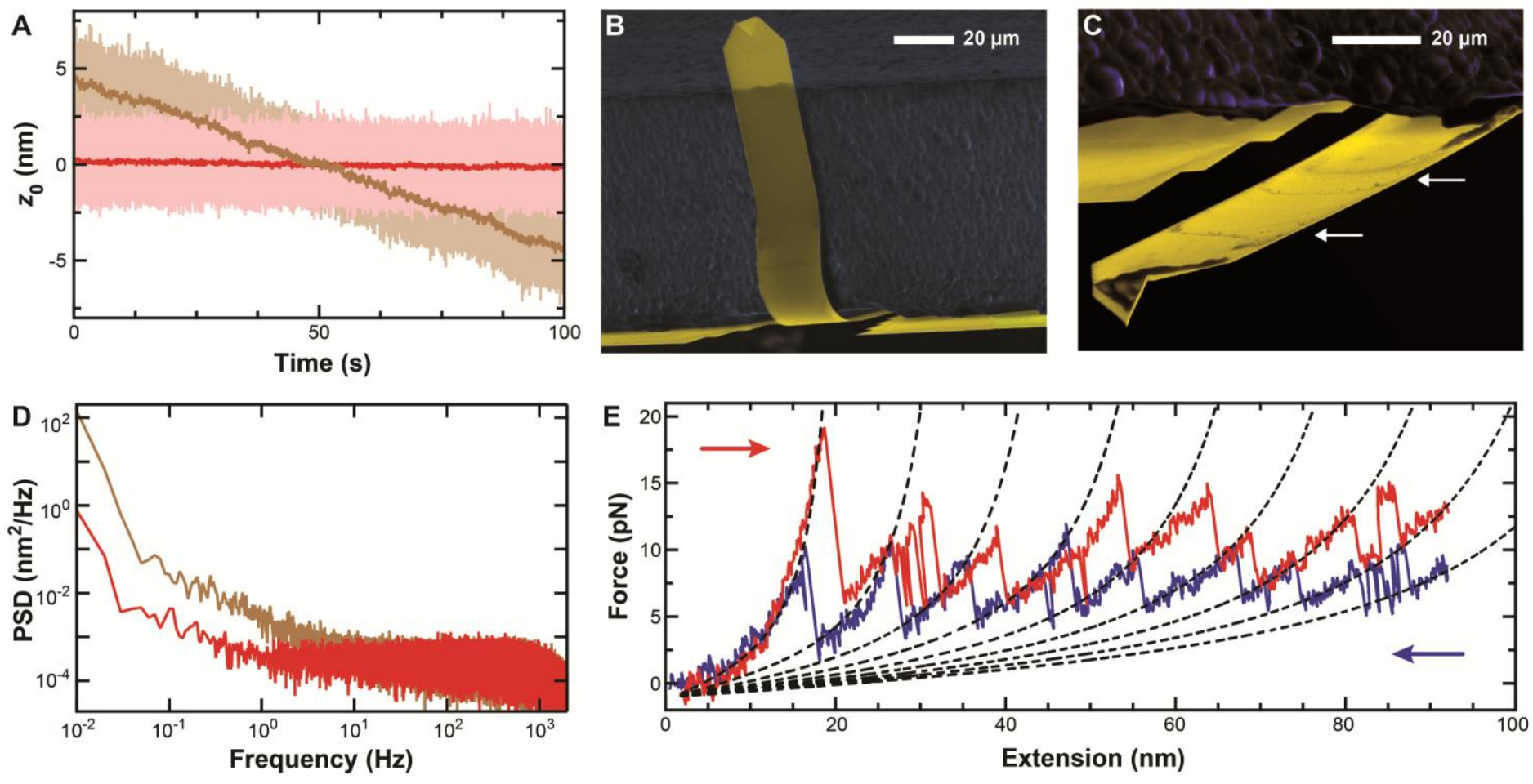

Fig. 5: Improved force stability with uncoated cantilevers. (A) The zero-force position $\left(z_{0}\right)$ of the cantilever plotted as a function of time for a gold-coated $(k=6.8 \mathrm{pN} / \mathrm{nm}$, gold $)$ and an uncoated $(k=7.1 \mathrm{pN} / \mathrm{nm}, \mathrm{red})$ cantilever after $>2 \mathrm{~h}$ in liquid. High-bandwidth data $(2.5 \mathrm{kHz})$ are shown in light colors and smoothed data $(10 \mathrm{~Hz})$ in dark colors. Note that, while the gold-coated cantilever has an approximately linear drift, there can be significant short-term fluctuations. (B) SEM image of a long BioLever stuck to the chip after wetting. (C) SEM of the same individual cantilever after unfolding. Damage to the gold coating can be seen in the unfolded image (white arrows). (D) Averaged PSDs of five consecutive 100-s records such as those shown in A. Panels A-D adapted from (Churnside et al., 2012). (E) A force-extension plot showing the nearequilibrium folding of a polyprotein consisting of 8 domains of $\mathrm{NuG} 2$ as the cantilever is retracted (red) and then subsequently brought towards (blue) the sample surface at $2 \mathrm{~nm} / \mathrm{s}$. Importantly, the low drift rate of the uncoated cantilever enables multiple folding/unfolding transitions of NuG2 to be observed. Worm-like-chain curves (black dashed lines) show the nominal opening distance for $\mathrm{NuG} 2$; small deviations from these curves are most likely due to residual drift in the cantilever or the AFM at this very low stretching velocity. Adapted from (He et al., 2015). 

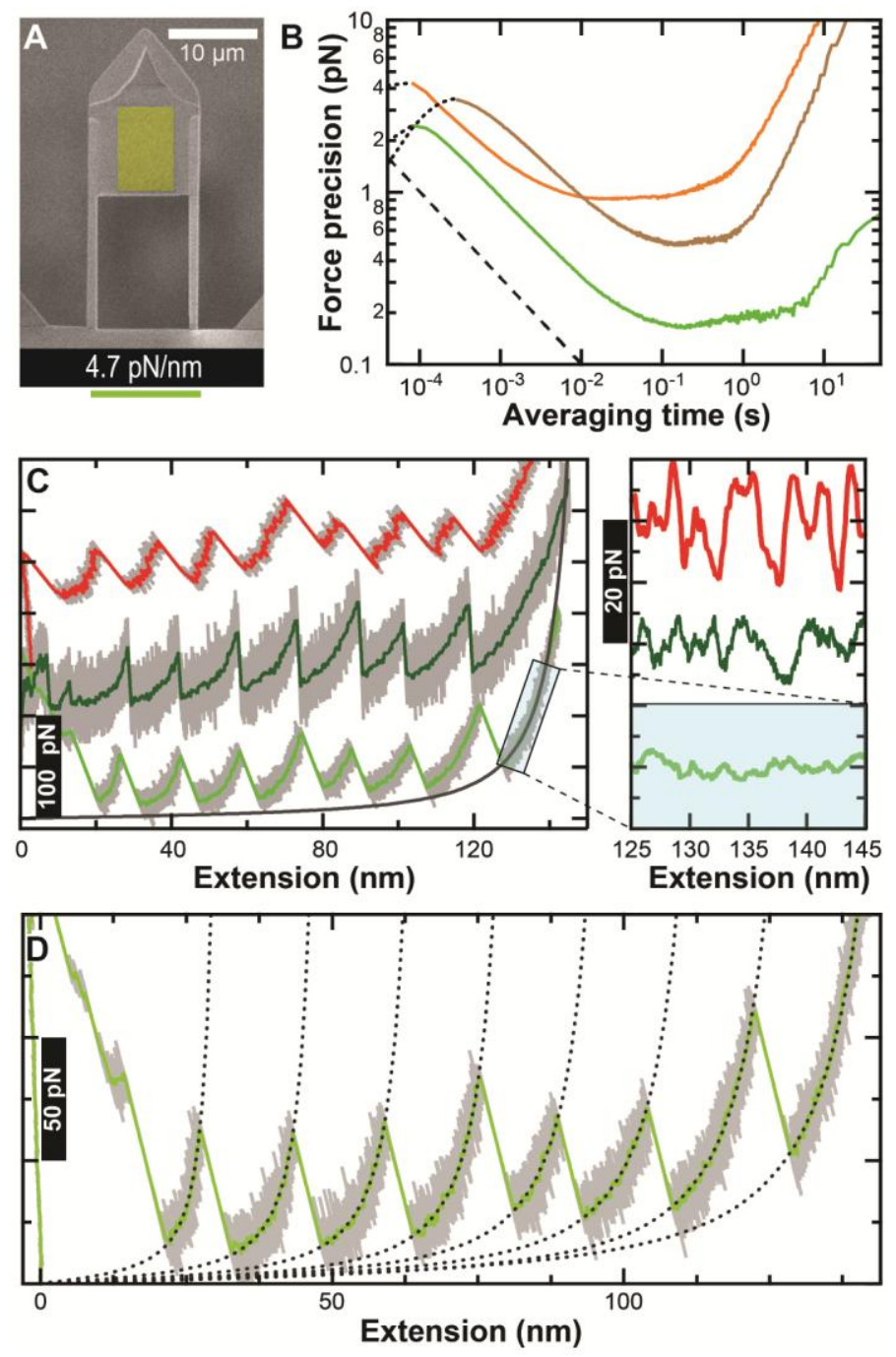

Fig. 6. FIB-modified BioLever Mini shows sub-pN force precision over a broad timescale (0.001-100 s). (A) SEM image of an FIB-modified BioLever Mini that has a transparent glasscapping layer protecting an underlying gold patch at the end of the cantilever. The cantilever's measured spring constant is indicated. The color of the line below the image is associated with curves in B-D. (B) Force precision plotted as a function of averaging time, technically the Allan deviation, calculated from a 100-s trace measured $50 \mathrm{~nm}$ over a surface for three highly reflective cantilevers [a gold-coated BioLever Mini ( $k=79 \mathrm{pN} / \mathrm{nm}$, orange), a gold-coated long BioLever $(k=5.0 \mathrm{pN} / \mathrm{nm}$, gold $)$, and a FIB-modified BioLever Mini $(k=5.1 \mathrm{pN} / \mathrm{nm}$, light green)]. The dashed line is a reference with a slope consistent with the averaging of Brownian motion. The FIB-modified cantilever significantly outperformed both commercial cantilevers, particularly on timescales longer than $0.1 \mathrm{~s}$. On these timescales, the detrimental effect of gold 
on force stability is particularly pronounced. We note that, at the very shortest times when the motion of the cantilever becomes correlated, the Allan deviation yields misleading results on force precision, and this region of the trace is de-emphasized using a dotted line. (C) Forceextension records of mechanically unfolding a polyprotein of NuG2 at $400 \mathrm{~nm} / \mathrm{s}$ with the long BioLever (red), the BioLever Mini (dark green), and the FIB-modified BioLever Mini (light green). High-bandwidth data $(50 \mathrm{kHz}$, grey) and smoothed data (1 kHz, colored) are shown. A worm-like-chain model (black line) well describes the stretching of the protein in one state. Inset: plot of residual force fluctuations after fitting a $20-\mathrm{nm}$ section of the $1-\mathrm{kHz}$ data to a WLC model. The standard deviations of these fluctuations are 5.2, 2.6, and $1.2 \mathrm{pN}$, respectively. Traces displaced vertically for clarity. (D) Force-extension curve of the unfolding of a polyprotein of $\mathrm{NuG} 2$ shows excellent agreement with WLC fits to the data. The force-extension traces are shown both at high bandwidth $(50 \mathrm{kHz}$, grey) and smoothed $(1 \mathrm{kHz}$, light green $)$. The average opening distance was $17.7 \mathrm{~nm}$, in quantitative agreement with the theoretical value of 18 $\mathrm{nm}$ based on a rise per amino acid (aa) of $0.36 \mathrm{~nm} / \mathrm{aa}$ and the $2.1-\mathrm{nm}$ distance between the ends of an individual NuG2 domain (Cao et al., 2007). Panels A-D reprinted with permission from (Bull et al., 2014). 

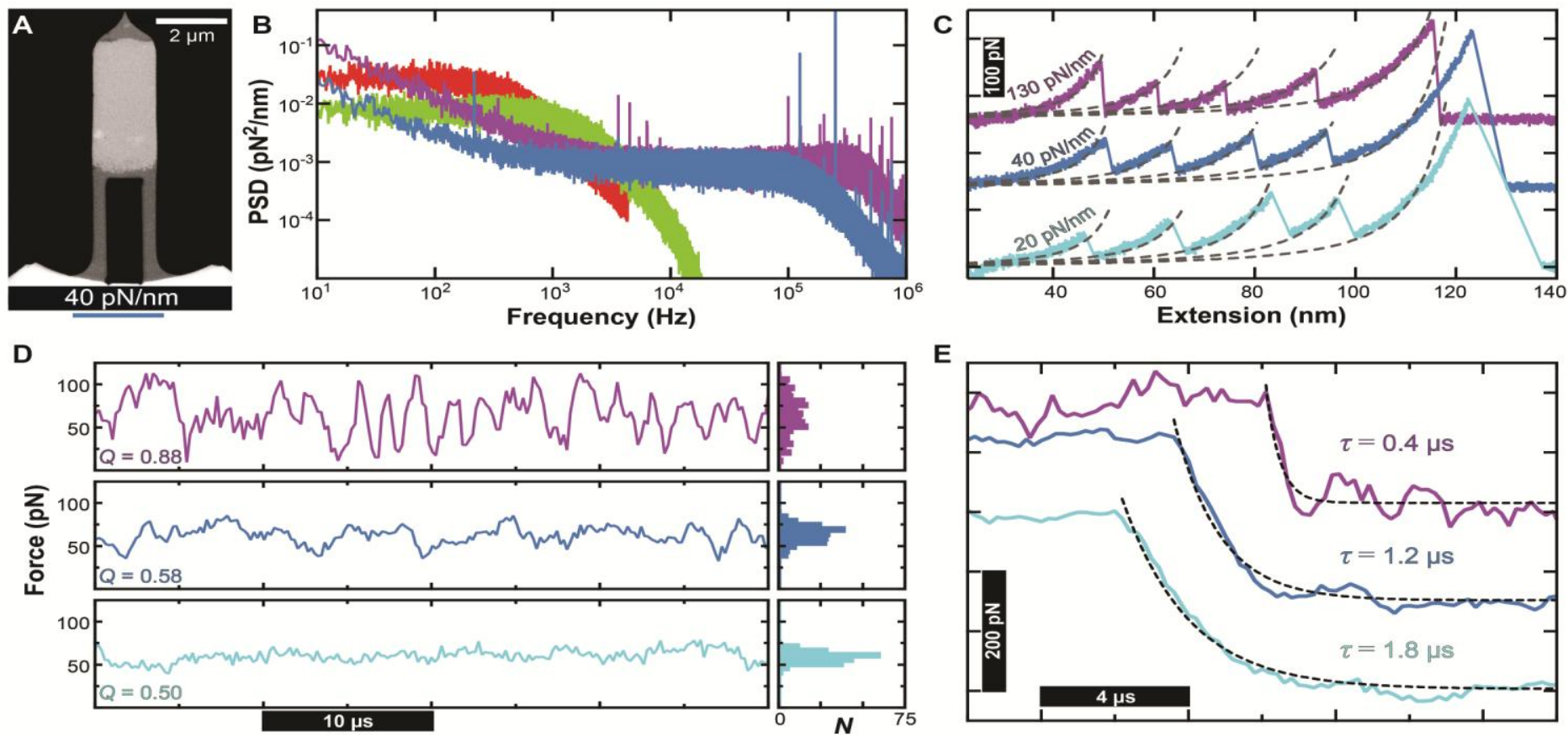

Fig. 7. Optimizing single-molecule force spectroscopy with $1-\mu$ s resolution using FIB-modified ultrashort cantilevers. (A) SEM images of an FIB-modified BioLever Fast. The cantilever's measured spring constant is indicated. The color of the line below the image is associated with curves in B-E. Reprinted with permission from (Edwards et al., 2015). (B) The force PSD is plotted as a function of frequency for an uncoated long BioLever $(L=100 \mu \mathrm{m}$, red); an FIBmodified BioLever Mini ( $L=40 \mu \mathrm{m}$, green), a gold-coated BioLever Fast $(L=9 \mu \mathrm{m}$, purple), and an FIB-modified BioLever Fast (blue). PSDs were taken at $50 \mathrm{~nm}$ over the surface. (C) Force-extension records showing the mechanical unfolding of the NuG2 polyprotein at $400 \mathrm{~nm} / \mathrm{s}$ with a standard BioLever Fast $(k=130 \mathrm{pN} / \mathrm{nm}$, purple $)$, a stiff modified BioLever Fast $(k=40$ $\mathrm{pN} / \mathrm{nm}$, blue $)$, and a soft modified BioLever Fast ( $k=20 \mathrm{pN} / \mathrm{nm}$, cyan). Data recorded at $50 \mathrm{kHz}$. Grey dashed lines represent worm-like-chain fits to the data, yielding a change in contour length $\left(\Delta L_{0}\right)$ of 17.3-17.9 nm, in agreement with previous results (Bull et al., 2014; Cao et al., 2007; He et al., 2015). Traces were laterally aligned and vertically displaced for clarity. (D) Force-versustime records while stretching the fully unfolded polyprotein at $\sim 60 \mathrm{pN}$. Histograms show the distribution in measured forces after a linear subtraction, with RMS deviations of 24, 11, and 6.8 $\mathrm{pN}$ for the purple, blue and cyan curves, respectively. Data recorded at $5 \mathrm{MHz}$. (E) Force-versustime record detailing the response of each cantilever used in $\mathrm{C}$ as the polyprotein detaches from 
the tip. Data recorded at $5 \mathrm{MHz}$. Time constants were determined by exponential fits (dashed black) to each record. B-E adapted from (Edwards et al., 2015). 


\section{References:}

Abbondanzieri, E.A., Greenleaf, W.J., Shaevitz, J.W., Landick, R., Block, S.M., 2005. Direct observation of base-pair stepping by RNA polymerase. Nature 438, 460-465.

Ando, T., 2012. High-speed atomic force microscopy coming of age. Nanotechnology 23, 062001.

Ando, T., Uchihashi, T., Kodera, N., 2013. High-speed AFM and applications to biomolecular systems. Annu. Rev. Biophys. 42, 393-414.

Ando, T., Uchihashi, T., Scheuring, S., 2014. Filming biomolecular processes by high-speed atomic force microscopy. Chem. Rev. 114, 3120-3188.

Bell, G.I., 1978. Models for specific adhesion of cells to cells. Science 200, 618-627.

Bippes, C.A., Muller, D.J., 2011. High-resolution atomic force microscopy and spectroscopy of native membrane proteins. Rep. Prog. Phys. 74, 086601.

Bull, M.S., Sullan, R.M., Li, H., Perkins, T.T., 2014. Improved single molecule force spectroscopy using micromachined cantilevers. ACS Nano 8, 4984-4995.

Cao, Y., Balamurali, M.M., Sharma, D., Li, H.B., 2007. A functional single-molecule binding assay via force spectroscopy. Proc. Natl. Acad. Sci. U.S.A. 104, 15677-15681.

Carrion-Vazquez, M., Oberhauser, A.F., Fowler, S.B., Marszalek, P.E., Broedel, S.E., Clarke, J., Fernandez, J.M., 1999. Mechanical and chemical unfolding of a single protein: A comparison. Proc. Natl. Acad. Sci. U.S.A. 96, 3694-3699.

Carter, A.R., Seol, Y., Perkins, T.T., 2009. Precision surface-coupled optical-trapping assays with 1 base-pair resolution. Biophys. J. 96, 2926-2934.

Cecconi, C., Shank, E.A., Bustamante, C., Marqusee, S., 2005. Direct observation of the threestate folding of a single protein molecule. Science 309, 2057-2060.

Chemla, Y.R., 2010. Revealing the base pair stepping dynamics of nucleic acid motor proteins with optical traps. Phys. Chem. Chem. Phys. 12, 3080-3095.

Chen, H., Yuan, G.H., Winardhi, R.S., Yao, M.X., Popa, I., Fernandez, J.M., Yan, J., 2015. Dynamics of equilibrium folding and unfolding transitions of titin immunoglobulin domain under constant forces. J. Am. Chem. Soc. 137, 3540-3546.

Chung, H.S., McHale, K., Louis, J.M., Eaton, W.A., 2012. Single-molecule fluorescence experiments determine protein folding transition path times. Science 335, 981-984. 
Churnside, A.B., Sullan, R.M., Nguyen, D.M., Case, S.O., Bull, M.S., King, G.M., Perkins, T.T., 2012. Routine and timely sub-picoNewton force stability and precision for biological applications of atomic force microscopy. Nano Lett. 12, 3557-3561.

Cossio, P., Hummer, G., Szabo, A., 2015. On artifacts in single-molecule force spectroscopy. Proc. Natl. Acad. Sci. U.S.A. 112, 14248-14253.

Czerwinski, F., Richardson, A.C., Oddershede, L.B., 2009. Quantifying noise in optical tweezers by Allan variance. Opt. Express 17, 13255-13269.

De Vlaminck, I., Henighan, T., van Loenhout, M.T., Pfeiffer, I., Huijts, J., Kerssemakers, J.W., Katan, A.J., van Langen-Suurling, A., van der Drift, E., Wyman, C., Dekker, C., 2011. Highly parallel magnetic tweezers by targeted DNA tethering. Nano Lett. 11, 5489-5493.

Doi, M., Edwards, S.E., 1986. The theory of polymer dynamics, Oxford Press, New York.

Dudko, O.K., Hummer, G., Szabo, A., 2006. Intrinsic rates and activation free energies from single-molecule pulling experiments. Phys. Rev. Lett. 96, 108101.

Dupres, V., Verbelen, C., Dufrene, Y.F., 2007. Probing molecular recognition sites on biosurfaces using AFM. Biomaterials 28, 2393-2402.

Edwards, D.T., Faulk, J.K., Sanders, A.W., Bull, M.S., Walder, R., LeBlanc, M.A., Sousa, M.C., Perkins, T.T., 2015. Optimizing 1- $\mu$ s-resolution single-molecule force spectroscopy on a commercial atomic force microscope. Nano Lett. 15, 7091-7098.

Evans, E., Ritchie, K., 1997. Dynamic strength of molecular adhesion bonds. Biophys. J. 72, 1541-1555.

Florin, E.-L., Moy, V.T., Gaub, H.E., 1994. Adhesion forces between individual ligand-receptor pairs. Science 264, 415-417.

Greenleaf, W.J., Woodside, M.T., Block, S.M., 2007. High-resolution, single-molecule measurements of biomolecular motion. Annu. Rev. Biophys. Biomol. 36, 171-190.

Greenleaf, W.J., Woodside, M.T., Abbondanzieri, E.A., Block, S.M., 2005. Passive all-optical force clamp for high-resolution laser trapping. Phys. Rev. Lett. 95, 208102.

He, C., Hu, C., Hu, X., Xiao, A., Perkins, T.T., Li, H., 2015. Direct observation of the reversible two-state unfolding and refolding of an alpha/beta protein by single-molecule atomic force microscopy. Angew. Chem. Int. Edit. 54, 9921-9925.

Herbert, K.M., Greenleaf, W.J., Block, S.M., 2008. Single-molecule studies of RNA polymerase: Motoring along. Annu. Rev. Biochem. 77, 149-176. 
Higgins, M.J., Proksch, R., Sader, J.E., Polcik, M., Mc Endoo, S., Cleveland, J.P., Jarvis, S.P., 2006. Noninvasive determination of optical lever sensitivity in atomic force microscopy. Rev. Sci. Instrum. 77, 013701.

Hodges, A.R., Bussmann, K.M., Hoh, J.H., 2001. Improved atomic force microscope cantilever performance by ion beam modification. Rev. Sci. Instrum. 72, 3880-3883.

Hoffmann, T., Dougan, L., 2012. Single molecule force spectroscopy using polyproteins. Chem. Soc. Rev. 41, 4781-4796.

Janovjak, H., Sapra, K.T., Kedrov, A., Muller, D.J., 2008. From valleys to ridges: exploring the dynamic energy landscape of single membrane proteins. ChemPhysChem. 9, 954-966.

Junker, J.P., Ziegler, F., Rief, M., 2009. Ligand-dependent equilibrium fluctuations of single calmodulin molecules. Science 323, 633-637.

Kienberger, F., Ebner, A., Gruber, H.J., Hinterdorfer, P., 2006. Molecular recognition imaging and force spectroscopy of single biomolecules. Acc. Chem. Res. 39, 29-36.

Kim, K., Saleh, O.A., 2009. A high-resolution magnetic tweezer for single-molecule measurements. Nucleic Acids Res. 37, e136.

King, G.M., Carter, A.R., Churnside, A.B., Eberle, L.S., Perkins, T.T., 2009. Ultrastable atomic force microscopy: atomic-scale lateral stability and registration in ambient conditions. Nano Lett. 9, 1451-1456.

Lee, G.U., Kidwell, D.A., Colton, R.J., 1994a. Sensing discrete streptavidin biotin interactions with atomic-force microscopy. Langmuir 10, 354-357.

Lee, G.U., Chrisey, L.A., Colton, R.J., 1994b. Direct measurement of the forces between complementary strands of DNA. Science 266, 771-773.

Lee, W., Zeng, X.C., Zhou, H.X., Bennett, V., Yang, W.T., Marszalek, P.E., 2010. Full reconstruction of a vectorial protein folding pathway by atomic force microscopy and molecular dynamics simulations. J. Biol. Chem. 285, 38167-38172.

Li, H., Cao, Y., 2010. Protein mechanics: from single molecules to functional biomaterials. Acc. Chem. Res. 43, 1331-1341.

Lindorff-Larsen, K., Piana, S., Dror, R.O., Shaw, D.E., 2011. How fast-folding proteins fold. Science 334, 517-520.

Liphardt, J., Onoa, B., Smith, S.B., Tinoco, I.J., Bustamante, C., 2001. Reversible unfolding of single RNA molecules by mechanical force. Science 292, 733-737. 
Long, X., Parks, J.W., Bagshaw, C.R., Stone, M.D., 2013. Mechanical unfolding of human telomere G-quadruplex DNA probed by integrated fluorescence and magnetic tweezers spectroscopy. Nucleic Acids Res. 41, 2746-2755.

Maali, A., Cohen-Bouhacina, T., Jai, C., Hurth, C., Boisgard, R., Aime, J.P., Mariolle, D., Bertin, F., 2006. Reduction of the cantilever hydrodynamic damping near a surface by ion-beam milling. J. Appl. Phys. 99, 024906.

Marszalek, P.E., Lu, H., Li, H., Carrion-Vazquez, M., Oberhauser, A.F., Schulten, K., Fernandez, J.M., 1999. Mechanical unfolding intermediates in titin modules. Nature 402, $100-103$.

Medalsy, I., Hensen, U., Muller, D.J., 2011. Imaging and quantifying chemical and physical properties of native proteins at molecular resolution by force-volume AFM. Angew. Chem., Int. Ed. 50, 12103-12108.

Meirovitch, L., 1997. Principles and techniques of tibrations, Prentice Hall, Upper Saddle River, NJ.

Merkel, R., Nassoy, P., Leung, A., Ritchie, K., Evans, E., 1999. Energy landscapes of receptorligand bonds explored with dynamic force spectroscopy. Nature 397, 50-53.

Moy, V.T., Florin, E.L., Gaub, H.E., 1994. Intermolecular forces and energies between ligands and receptors. Science 266, 257-259.

Muller, D.J., Dufrene, Y.F., 2008. Atomic force microscopy as a multifunctional molecular toolbox in nanobiotechnology. Nat. Nanotechnol. 3, 261-269.

Nam, G.M., Makarov, D.E., 2016. Extracting intrinsic dynamic parameters of biomolecular folding from single-molecule force spectroscopy experiments. Protein Sci. 25, 123-134.

Neuman, K.C., Nagy, A., 2008. Single-molecule force spectroscopy: optical tweezers, magnetic tweezers and atomic force microscopy. Nat. Methods 5, 491-505.

Oesterhelt, F., Oesterhelt, D., Pfeiffer, M., Engel, A., Gaub, H.E., Muller, D.J., 2000. Unfolding pathways of individual bacteriorhodopsins. Science 288, 143-146.

Onuchic, J.N., Luthey-Schulten, Z., Wolynes, P.G., 1997. Theory of protein folding: the energy landscape perspective. Annu. Rev. Phys. Chem. 48, 545-600.

Otten, M., Ott, W., Jobst, M.A., Milles, L.F., Verdorfer, T., Pippig, D.A., Nash, M.A., Gaub, H.E., 2014. From genes to protein mechanics on a chip. Nat. Methods 11, 1127-1130.

Paolino, P., Bellon, L., 2009. Frequency dependence of viscous and viscoelastic dissipation in coated micro-cantilevers from noise measurement. Nanotechnology 20, 405705. 
Popa, I., Berkovich, R., Alegre-Cebollada, J., Badilla, C.L., Rivas-Pardo, J.A., Taniguchi, Y., Kawakami, M., Fernandez, J.M., 2013. Nanomechanics of HaloTag tethers. J. Am. Chem. Soc. 135, 12762-12771.

Puchner, E.M., Gaub, H.E., 2009. Force and function: probing proteins with AFM-based force spectroscopy. Curr. Opin. Struct. Biol. 19, 605-614.

Quake, S.R., Babcock, H., Chu, S., 1997. The dynamics of partially extended single molecules of DNA. Nature 388, 151-154.

Radmacher, M., Cleveland, J.P., Hansma, P.K., 1995. Improvement of thermally induced bending of cantilevers used for atomic-force microscopy. Scanning 17, 117-121.

Rico, F., Su, C.M., Scheuring, S., 2011. Mechanical mapping of single membrane proteins at submolecular resolution. Nano Lett. 11, 3983-3986.

Rico, F., Gonzalez, L., Casuso, I., Puig-Vidal, M., Scheuring, S., 2013. High-speed force spectroscopy unfolds titin at the velocity of molecular dynamics simulations. Science $342,741-743$.

Rief, M., Gautel, M., Oesterhelt, F., Fernandez, J.M., Gaub, H.E., 1997. Reversible unfolding of individual titin immunoglobulin domains by AFM. Science 276, 1109-1112.

Sader, J.E., Chon, J.W.M., Mulvaney, P., 1999. Calibration of rectangular atomic force microscope cantilevers. Rev. Sci. Instrum. 70, 3967-3969.

Sader, J.E., Sanelli, J.A., Adamson, B.D., Monty, J.P., Wei, X.Z., Crawford, S.A., Friend, J.R., Marusic, I., Mulvaney, P., Bieske, E.J., 2012. Spring constant calibration of atomic force microscope cantilevers of arbitrary shape. Rev. Sci. Instrum. 83, 103705.

Sahin, O., Erina, N., 2008. High-resolution and large dynamic range nanomechanical mapping in tapping-mode atomic force microscopy. Nanotechnology 19, 445717.

Sapra, K.T., Besir, H., Oesterhelt, D., Muller, D.J., 2006. Characterizing molecular interactions in different bacteriorhodopsin assemblies by single-molecule force spectroscopy. J. Mol. Biol. 355, 640-650.

Scheuring, S., Dufrene, Y.F., 2010. Atomic force microscopy: probing the spatial organization, interactions and elasticity of microbial cell envelopes at molecular resolution. Mol. Microbiol. 75, 1327-1336.

Schuler, B., Hofmann, H., 2013. Single-molecule spectroscopy of protein folding dynamicsexpanding scope and timescales. Curr. Opin. Struc. Biol. 23, 36-47.

Shaevitz, J.W., Abbondanzieri, E.A., Landick, R., Block, S.M., 2003. Backtracking by single RNA polymerase molecules observed at near-base-pair resolution. Nature 426, 684-687. 
Smith, S.B., Finzi, L., Bustamante, C., 1992. Direct mechanical measurements of the elasticity of single DNA molecules by using magnetic beads. Science 258, 1122-1126.

Soranno, A., Buchli, B., Nettels, D., Cheng, R.R., Muller-Spath, S., Pfeil, S.H., Hoffmann, A., Lipman, E.A., Makarov, D.E., Schuler, B., 2012. Quantifying internal friction in unfolded and intrinsically disordered proteins with single-molecule spectroscopy. Proc. Natl. Acad. Sci. U.S.A. 109, 17800-17806.

Stahl, S.W., Nash, M.A., Fried, D.B., Slutzki, M., Barak, Y., Bayer, E.A., Gaub, H.E., 2012. Single-molecule dissection of the high-affinity cohesin-dockerin complex. Proc. Natl. Acad. Sci. USA 109, 20431-20436.

Stigler, J., Ziegler, F., Gieseke, A., Gebhardt, J.C.M., Rief, M., 2011. The complex folding network of single calmodulin molecules. Science 334, 512-516.

Sullan, R.M., Churnside, A.B., Nguyen, D.M., Bull, M.S., Perkins, T.T., 2013. Atomic force microscopy with sub-picoNewton force stability for biological applications. Methods 60, $131-141$

Sullivan, D.B., Allan, D.W., Howe, D.A., Walls, E.L. 1990. Characterization of Clocks and Oscillators, National Institute of Standards and Technology Technical Note 1337. U.S. Government Printing Office.

Taniguchi, Y., Kawakami, M., 2010. Application of HaloTag protein to covalent immobilization of recombinant proteins for single molecule force spectroscopy. Langmuir 26, 1043310436.

Viani, M.B., Schaffer, T.E., Chand, A., Rief, M., Gaub, H.E., Hansma, P.K., 1999. Small cantilevers for force spectroscopy of single molecules. J. Appl. Phys. 86, 2258-2262.

Woodside, M.T., Behnke-Parks, W.M., Larizadeh, K., Travers, K., Herschlag, D., Block, S.M., 2006a. Nanomechanical measurements of the sequence-dependent folding landscapes of single nucleic acid hairpins. Proc. Natl. Acad. Sci. U.S.A. 103, 6190-6195.

Woodside, M.T., Anthony, P.C., Behnke-Parks, W.M., Larizadeh, K., Herschlag, D., Block, S.M., 2006b. Direct measurement of the full, sequence-dependent folding landscape of a nucleic acid. Science 314, 1001-1004.

Yu, H., Gupta, A.N., Liu, X., Neupane, K., Brigley, A.M., Sosova, I., Woodside, M.T., 2012a. Energy landscape analysis of native folding of the prion protein yields the diffusion constant, transition path time, and rates. Proc. Natl. Acad. Sci. U.S.A. 109, 14452-14457.

Yu, H., Liu, X., Neupane, K., Gupta, A.N., Brigley, A.M., Solanki, A., Sosova, I., Woodside, M.T., 2012b. Direct observation of multiple misfolding pathways in a single prion protein molecule. Proc. Natl. Acad. Sci. U.S.A. 109, 5283-5288. 
Zakeri, B., Fierer, J.O., Celik, E., Chittock, E.C., Schwarz-Linek, U., Moy, V.T., Howarth, M., 2012. Peptide tag forming a rapid covalent bond to a protein, through engineering a bacterial adhesin. Proc. Nat. Acad. Sci. U.S.A. 109, E690-E697.

Zhang, S., Aslan, H., Besenbacher, F., Dong, M., 2014. Quantitative biomolecular imaging by dynamic nanomechanical mapping. Chem. Soc. Rev. 43, 7412-7429.

Zimmermann, J.L., Nicolaus, T., Neuert, G., Blank, K., 2010. Thiol-based, site-specific and covalent immobilization of biomolecules for single-molecule experiments. Nat. Protoc. 5, 975-985.

Zoldak, G., Rief, M., 2013. Force as a single molecule probe of multidimensional protein energy landscapes. Curr. Opin. Struc. Biol. 23, 48-57.

Zoldak, G., Stigler, J., Pelz, B., Li, H., Rief, M., 2013. Ultrafast folding kinetics and cooperativity of villin headpiece in single-molecule force spectroscopy. Proc. Natl. Acad. Sci. U.S.A. 110, 18156-18161. 

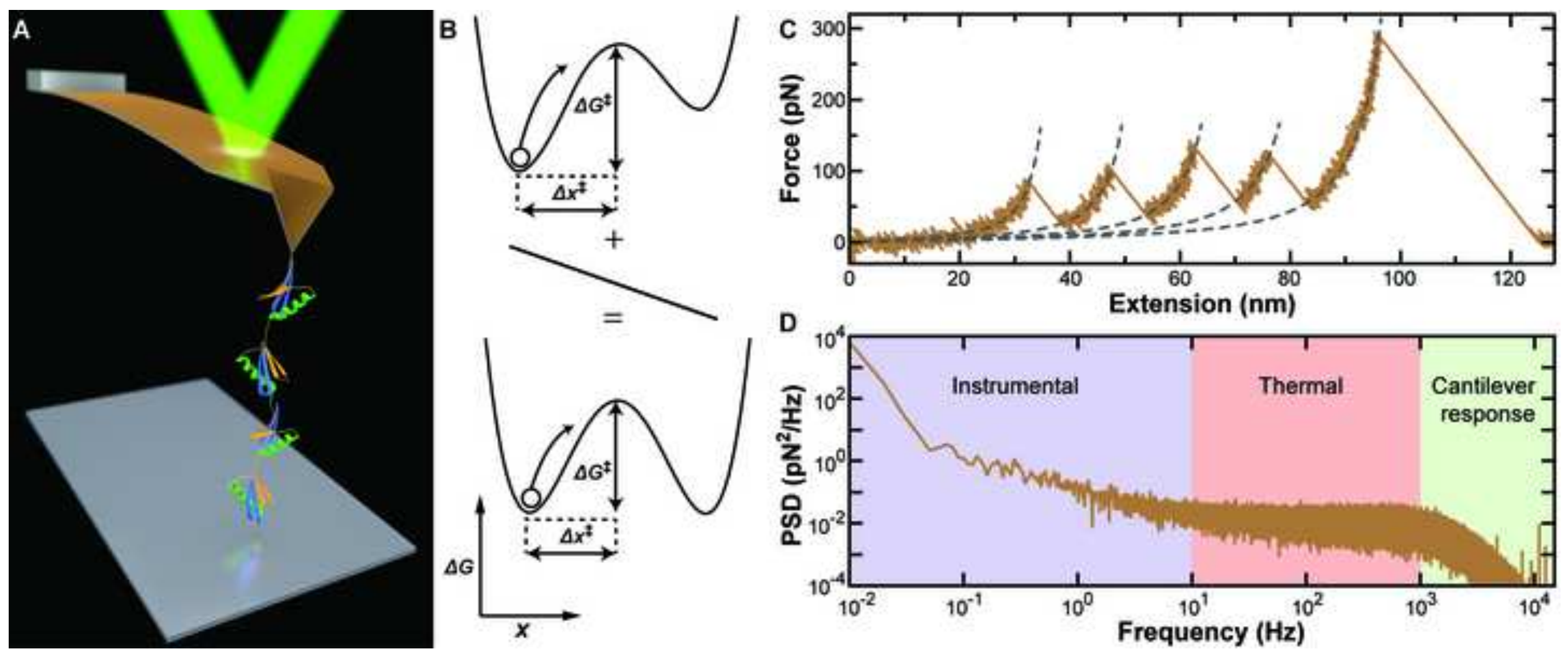


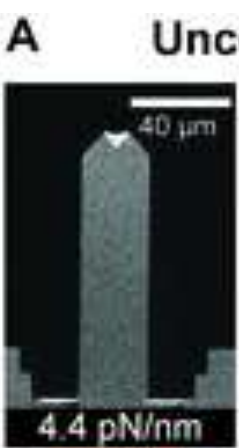

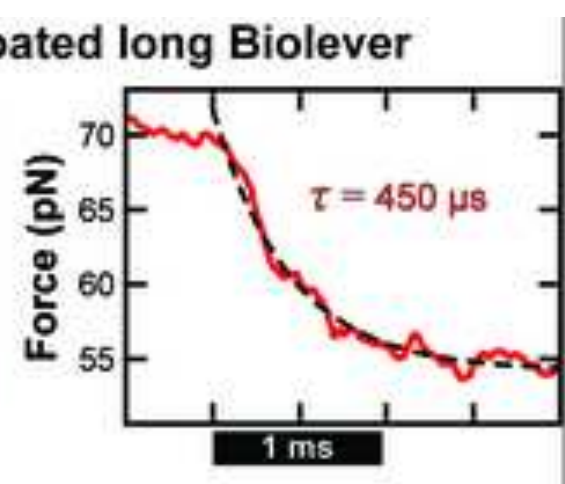

\section{Pros: - Excellent force stability} -Easy to prepare

\section{Cons: $\cdot$ Moderate temporal resolution} - Moderate force precision

- Low reflectivity

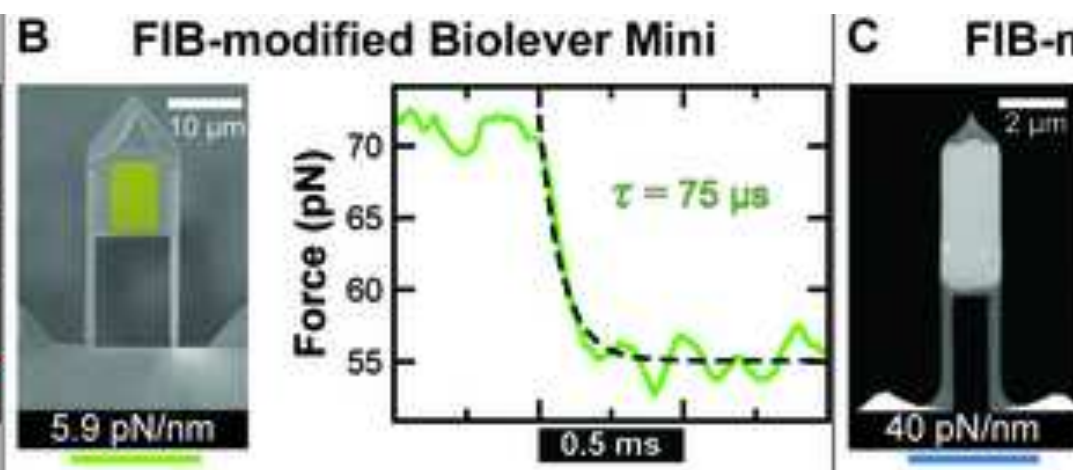

FIB-modified Biolever Fast

Pros: - Excellent force stability - Good force precision - Good temporal resolution - High reflectivity

Cons: · Requires FIB modification
Pros: - Excellent temporal resolution - Excellent force precision

- Avoids cantilever ringing

Cons: - Requires FIB modification - Requires custom detection laser - Moderate stability 

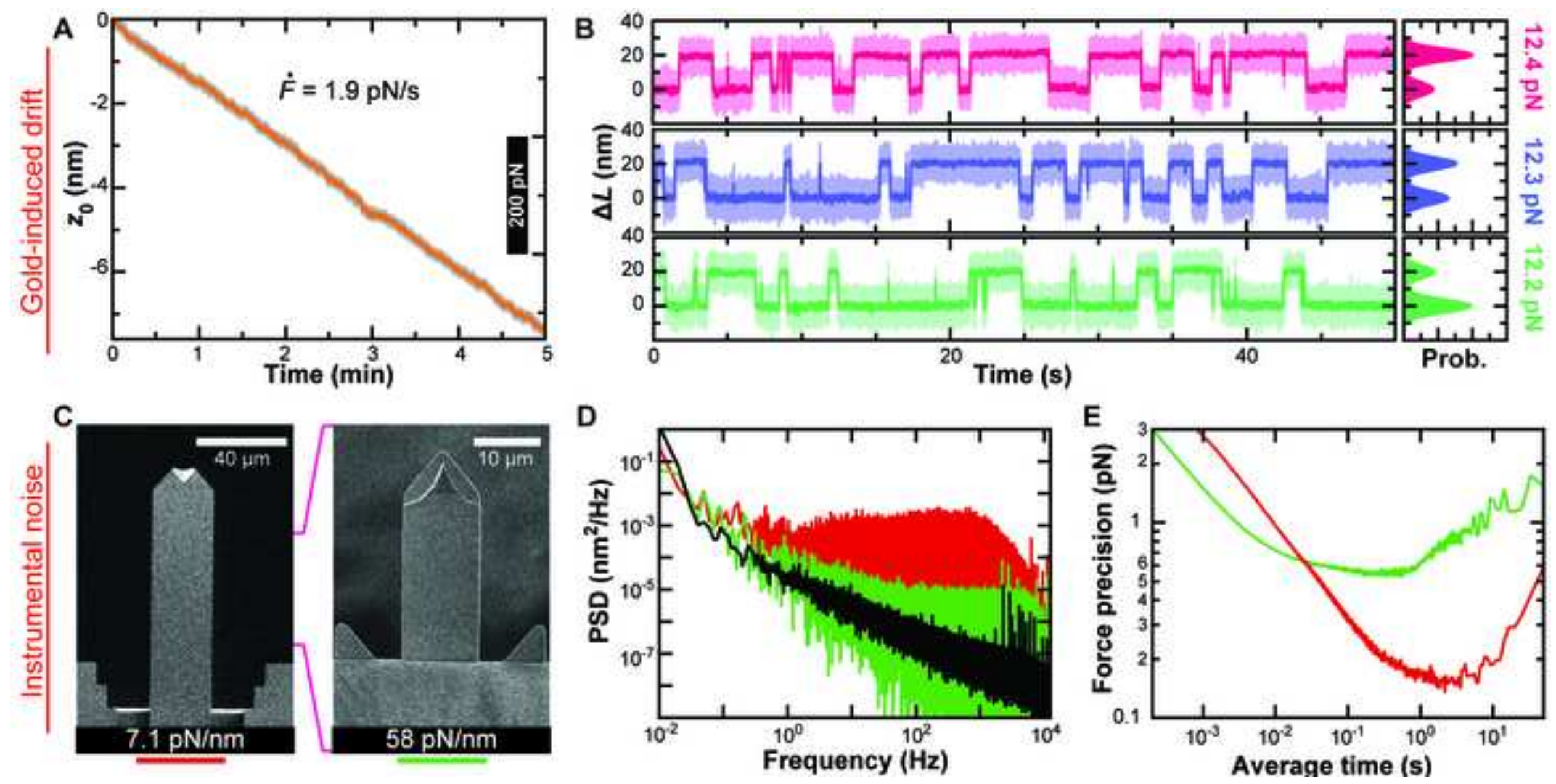

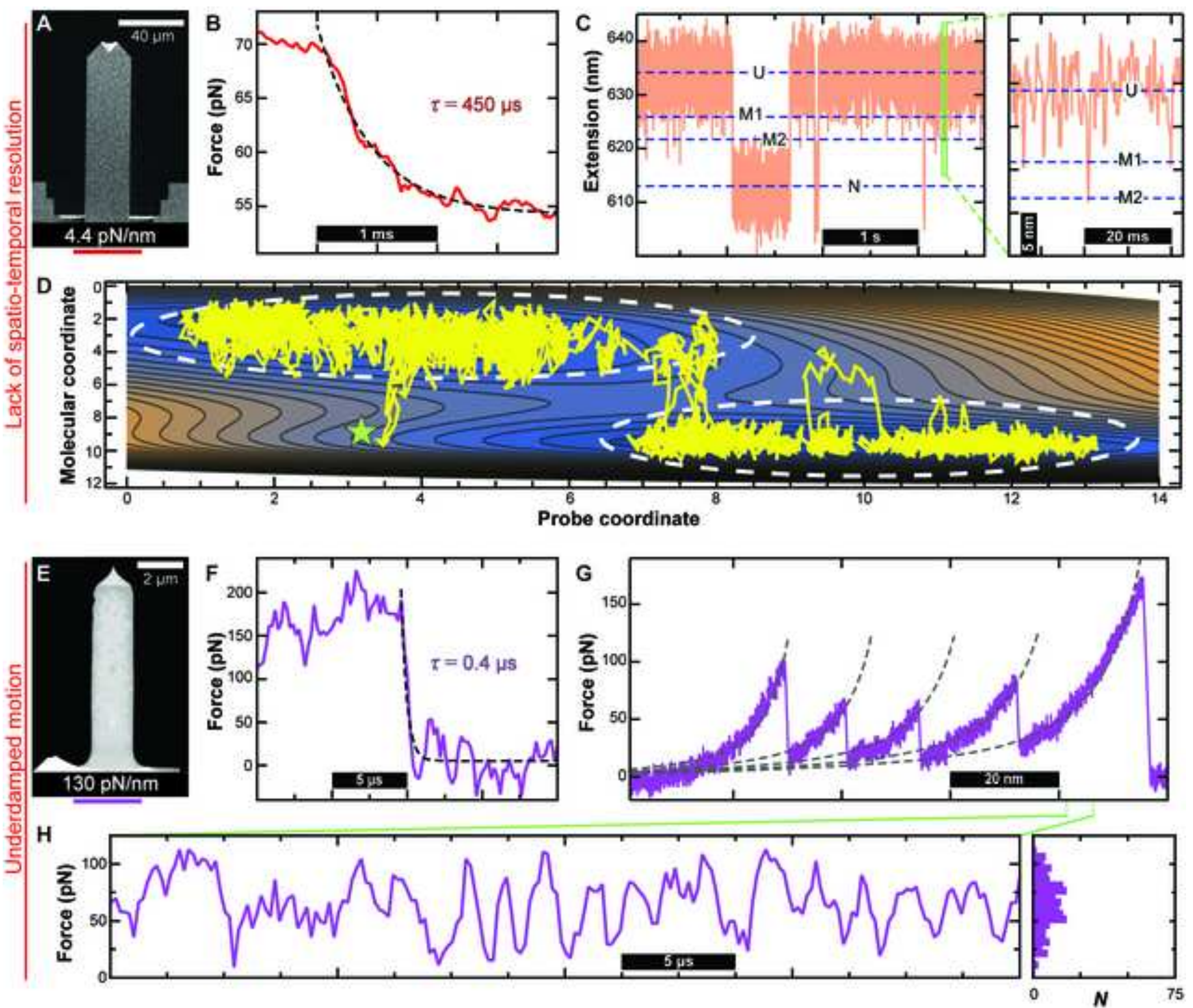

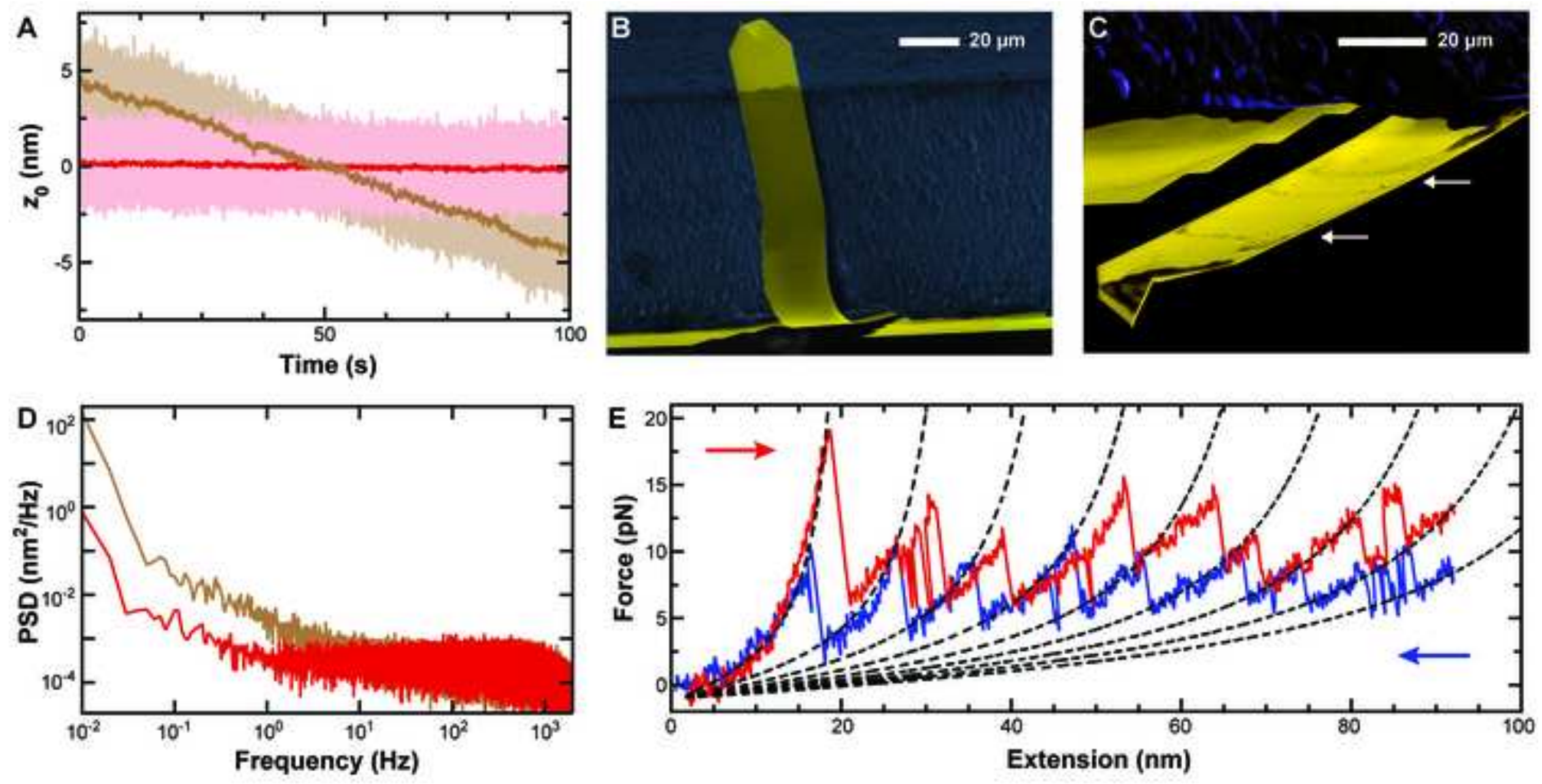

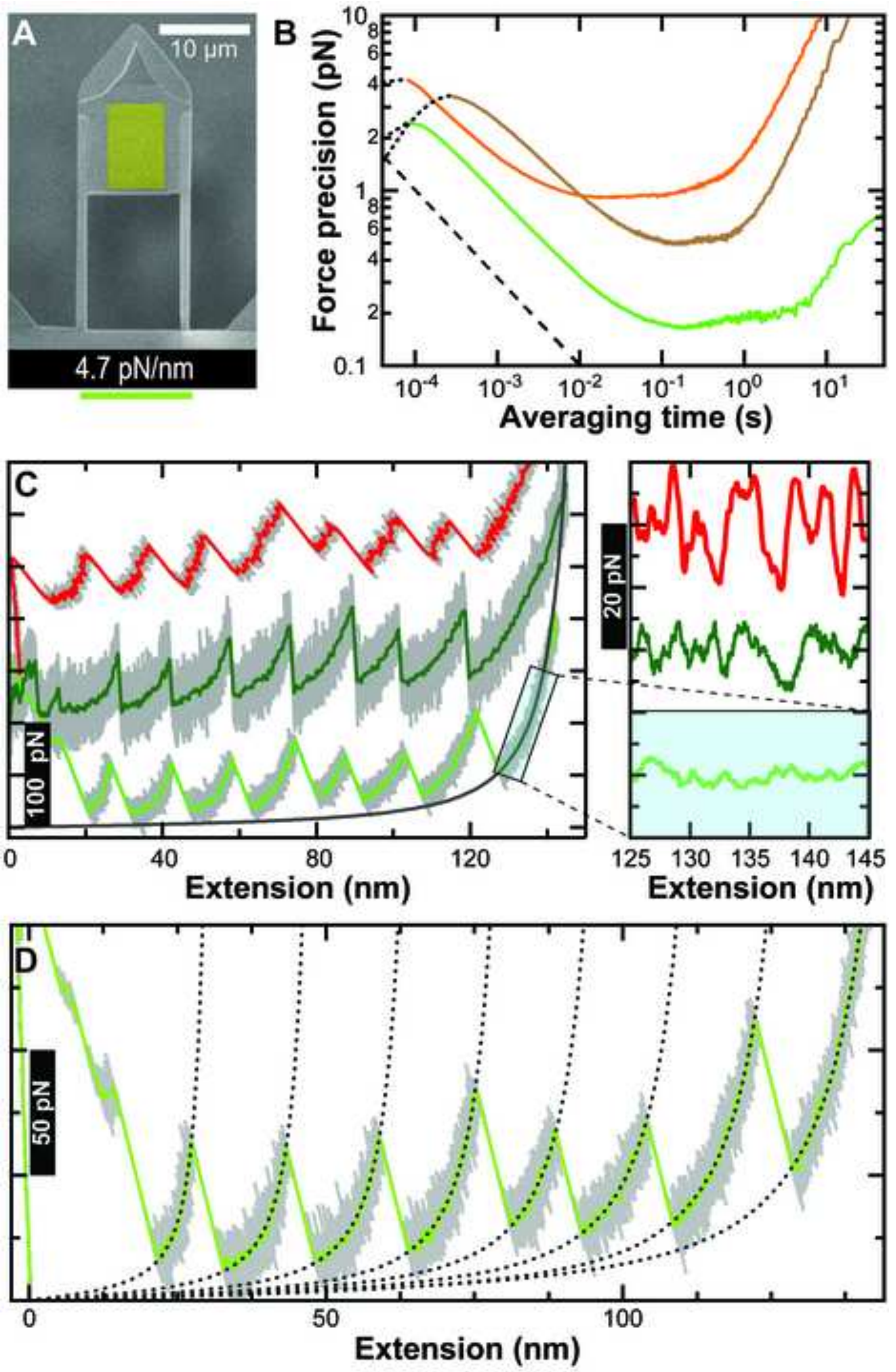


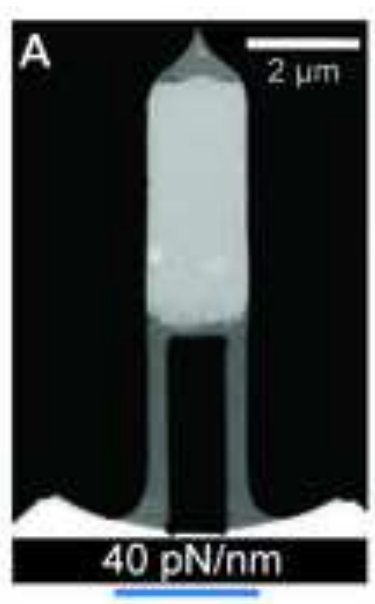

D
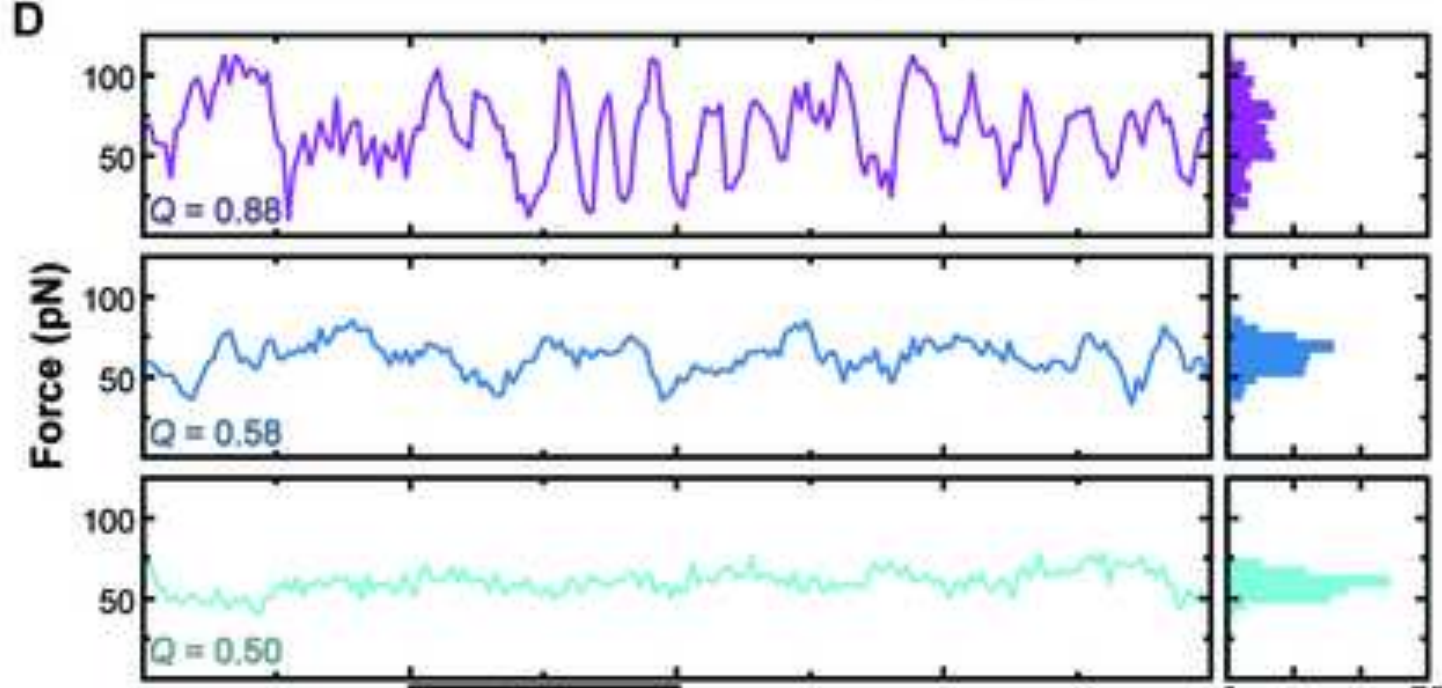

$10 \mu \mathrm{s}$
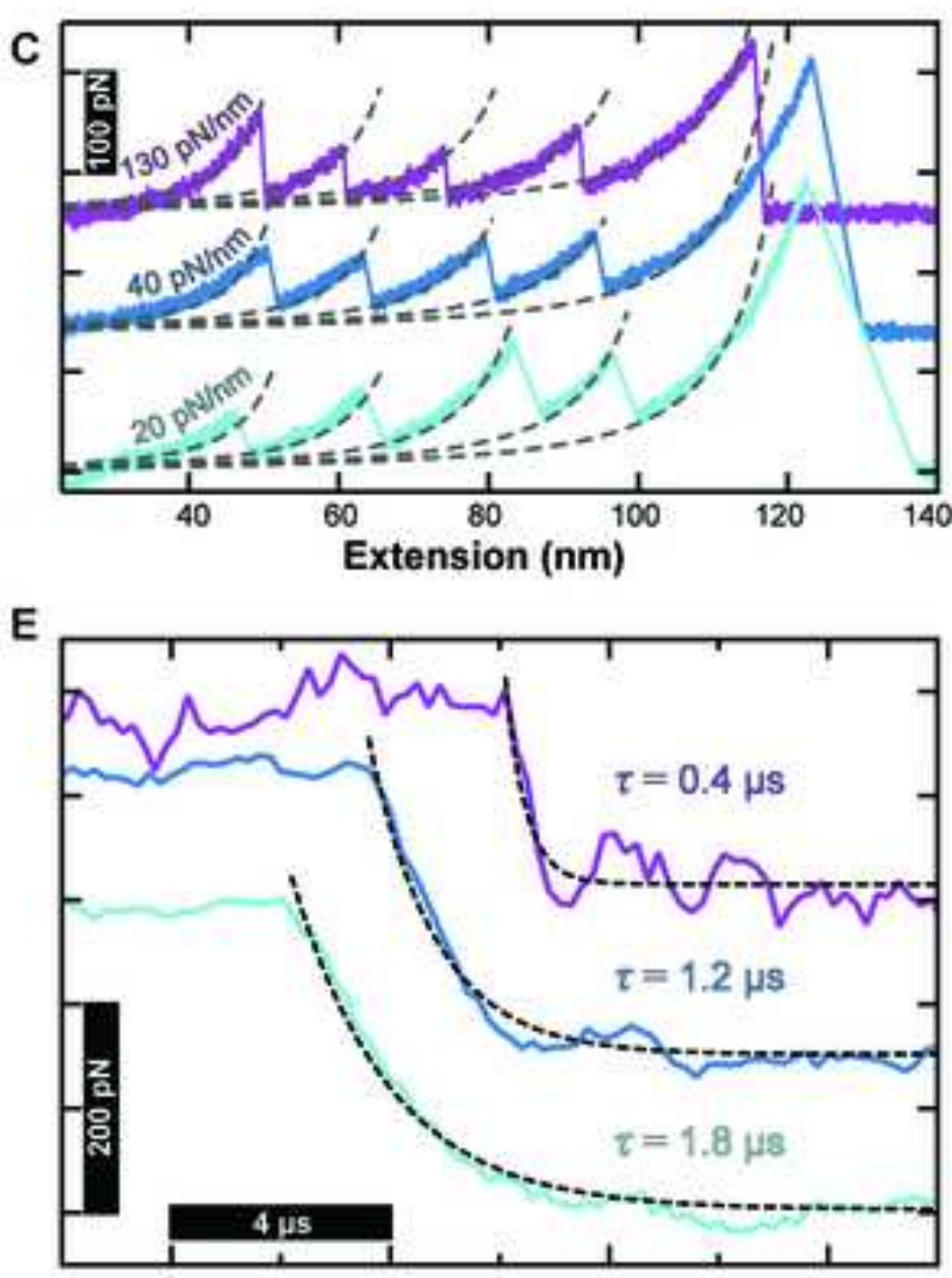